\title{
Development, Analysis and Applications of a Quantitative Methodology for Assessing Customer Satisfaction using Evolutionary Optimization
}

\author{
Sunith Bandaru ${ }^{\mathrm{a}}$, Abhinav Gaur ${ }^{\mathrm{b}}$, Kalyanmoy Deb ${ }^{\mathrm{b}}$, \\ Vineet Khare $^{\mathrm{c}}$, Rahul Chougule ${ }^{\mathrm{d}}$, Pulak Bandyopadhyay ${ }^{\mathrm{e}}$ \\ ${ }^{a}$ Virtual Systems Research Centre, University of Skövde, Skövde 54128, Sweden \\ sunith.bandaru@his.se \\ ${ }^{b}$ Department of Electrical and Computer Engineering, Michigan State University, \\ East Lansing, MI 48824,USA \\ \{gaurabhi@msu.edu,kdeb@egr.msu.edu\} \\ ${ }^{c}$ Amazon Development Centre (India) Pvt. Ltd., Bengaluru 560055, India \\ (Previously with India Science Lab, General Motors Global R\&D, Bangalore, India) \\ vkhare@amazon.com \\ ${ }^{d}$ Department of Mechanical Engineering, Walchand College of Engineering, \\ Sangli, Maharashtra 416415, India \\ (Previously with India Science Lab, General Motors Global R\&D, Bangalore, India) \\ chougulerahulg@gmail.com \\ ${ }^{e}$ General Motors RED Center, Warren, MI 48090, USA \\ (Retired from General Motors, Consultant) \\ Pulak_b@hotmail.com
}

\begin{abstract}
Consumer-oriented companies are getting increasingly more sensitive about customer's perception of their products, not only to get a feedback on their popularity, but also to improve the quality and service through a better understanding of design issues for further development. However, a consumer's perception is often qualitative and is achieved through third party surveys or the company's recording of after-sale feedback through explicit surveys or warranty based commitments. In this paper, we consider an automobile company's warranty records for different vehicle models and suggest a data mining procedure to assign a customer satisfaction index (CSI) to each vehicle model based on the perceived notion of the level of satisfaction of customers. Based on the developed CSI function, customers are then divided into satisfied and dissatisfied customer groups. The warranty data are then clustered separately for each group and analyzed to find possible causes (field failures) and their relative effects on customer's satisfaction (or dissatisfaction) for a vehicle model. Finally, speculative introspection
\end{abstract}


has been made to identify the amount of improvement in CSI that can be achieved by the reduction of some critical field failures through better design practices. Thus, this paper shows how warranty data from customers can be utilized to have a better perception of ranking of a product compared to its competitors in the market and also to identify possible causes for making some customers dissatisfied and eventually to help percolate these issues at the design level. This closes the design cycle loop in which after a design is converted into a product, its perceived level of satisfaction by customers can also provide valuable information to help make the design better in an iterative manner. The proposed methodology is generic and novel, and can be applied to other consumer products as well.

Keywords: Customer Satisfaction Index (CSI), quantitative modeling, evolutionary optimization, Customer Relationship Management (CRM)

\section{Introduction}

Most companies spend a considerable amount of effort in improving their products from a technical point of view. Although it is right on its own merit, today's advancement in computing algorithms and fast computing hardware enable companies to obtain a more direct feedback on their products from their own customers. When a company produces a range of products (such as a platform of designs) with different trade-offs between cost, quality, and environmental effects, the level of satisfaction of one product over the other as perceived by customers bears a wealth of information about 'why a product is liked or not liked by users?'. Interestingly, such information, if derived from after-sale surveys or warranty data, can provide valuable information to the designers for a possible improvement of the product for the next design phase. Since this is somewhat intuitive and importantly many successful companies do collect and record warranty or other after-sales information, it is now a matter of a research study to devise a mechanism to retrieve hidden information from the data. In this paper, we make an effort in this direction using an automobile industry's warranty data to demonstrate the procedure.

Customer satisfaction has been defined in the literature as 'the state of mind that customers have about a company when their expectations have been met or exceeded over the lifetime of the product or service' $[1,2]$. Customer satisfaction leads to customer retention, customer loyalty and product repurchase. Thus its measurement is an important and integral part of an effective Customer Relationship Management (CRM). Broadly speaking, 
satisfaction measures involve three psychological elements for evaluation of the product or service experience: (i) cognitive, which depends on the actual use of the product or service by the customer, (ii) affective, which depends on the customer's attitude towards the product or service or the company and (iii) behavioral, which depends on the customer's view regarding another product or service from the same company [3]. An important implication of the above definition for customer satisfaction is that it is subjective. Due to its non-quantifiability, most companies resort to a survey/questionnaire based assessment for the measurement of their products' perceived satisfaction. In this regard, years of research on customer behavior has led to specification of ten domains of satisfaction, ranging from quality, efficiency, etc. to commitment to the customer and product innovation [4]. Surveys are designed to touch these domains. What actually to ask in the survey depends on the kind of product or service provided, the kinds of customers served, number of customers served, the longevity and frequency of customer/supplier interactions, and what is to be done with the results. The very nature of such surveys requires the customer to evaluate each statement on a psychometric scale (or a rating scale) [5]. Likert scales [6], semantic differential scales [7], smiling faces scales and percentage measures [8] are popular in that order. A typical five-level Likert item consists of a statement and asks the surveyee to choose among (a) Strongly disagree, (b) Disagree, (c) Neutral, (d) Agree, and (e) Strongly agree. Several studies exist which show the merit and demerits of different rating scales and how they should be interpreted.

In the automotive sector, Original Equipment Manufacturers (OEMs) depend on reports published by various marketing information firms, like the American Customer Satisfaction Index [9], J.D.Power and Associates [10] and Consumer Reports [11] for gaining insights into vehicle quality. Quality analysis data provided are often focused on questions related to number of failures in the field (namely, Incidents Per Thousand Vehicles (IPTV) [12] and Problems Per Hundred vehicles (PPH)) for individual components like engine, transmission, etc. Limited emphasis is placed on the assessment of individual users' perception and satisfaction resulting from day to day use of the product. Moreover, survey based estimates rely on a small sample of the customers (around 200 to 400 per vehicle model [10]). Despite this, the surveys themselves are highly regarded and play a significant role in molding the customer's attitude towards a particular vehicle model. To some extent, the surveys also help the OEMs in identifying major problem areas. The CRM policy of OEMs should therefore be flexible enough to take into account the information contained in these survey reports published 
annually.

For service based companies, Parasuraman et. al. [13, 14] proposed the 'gaps model' for estimating satisfaction objectively by using the gap between the customer's expectation and perceived experience of performance. Apart from these and a few other related studies, quantitative measurement of customer satisfaction has not received much attention in the literature. The main reason is as follows: There are three practical approaches to measuring satisfaction, namely, post-purchase evaluation, periodic satisfaction surveys and continuous satisfaction tracking. Post-purchase evaluation (known as Initial Quality Study in the automotive sector) deals with satisfaction assessment shortly after the delivery of product or service. Periodic satisfaction surveys provide occasional snapshots of customer perceptions. Continuous satisfaction tracking is much like post-purchase evaluation but carried out over time. Post-purchase evaluations are very common and seems to be used across all sectors. Most products and services are however, not amenable to periodic assessment and therefore not enough data is obtained for a single customer to warrant a quantitative study.

Automotive OEMs, on the other hand, provide customers a warranty period which covers repairs and mechanical faults as part of the sale. Claims can be made by the customers at authorized dealerships and service stations which keep customer-specific records of these claims. Warranty data consists of claims data and supplementary data. A review of warranty data analysis methods for identifying early warnings of abnormalities in products, providing useful information about failure modes to aid design modification, estimating product reliability for deciding on warranty policy, and forecasting future warranty claims needed for preparing fiscal plans can be found in [15]. In this paper, we go a step further and use the same warranty data for obtaining a mathematical model for predicting customer satisfaction. Typically, customer satisfaction is measured at the individual level, but it is almost always reported at an aggregate level. We ensure that this is true for our model by employing a bottom-up approach to modeling. To illustrate, Table 1 shows some basic statistics of the five vehicle models considered in this study. The numbers correspond to the warranty data of all vehicles serviced between January 2008 and August 2009. For anonymity, the total number of customers and claims for each model are shown relative to those of the model with the lowest customer base (Model 5). The last row of the table shows the number of unique field failures, a common constituent of IPTV or $\mathrm{PPH}$ figures, that occurred in a vehicle model during the said period. A field failure refers to any vehicle-related problem faced by the customer for which he/she had to visit a dealer or service station. Each unique field failure is as- 
sociated with a corresponding repair code for classification purposes. Given the limited resources available with customer relation managers, it is only prudent to prioritize these field failures for a subsequent root-cause analysis and possible reduction in the next design phase. The methodology presented in this paper allows one to prioritize these unique field failures based on a quantitative measure of their potential for improvement in the customer's perception and hence the CSI. The method begins with the building of a quantitative model of the customer satisfaction index using an evolutionary optimization technique. The present work suggests an improvement to the method in [16] and validates it against Consumer Reports ratings of the vehicle models. The resulting CSI modeling function is then used to obtain the CSI Improvement Potential (CIP) for different types of field failures.

Table 1: Some basic statistics of the vehicle models relative to Model 5

\begin{tabular}{|c|c|c|c|c|c|}
\cline { 2 - 6 } \multicolumn{1}{c|}{} & \multicolumn{5}{c|}{ Vehicle Model Number } \\
\cline { 2 - 6 } \multicolumn{1}{c|}{} & 1 & 2 & 3 & 4 & 5 \\
\hline Segment & Compact & Midsize & Luxury & Midsize & Luxury \\
\hline Total Customers & $19.61 \times \mathrm{C}$ & $13.35 \times \mathrm{C}$ & $1.93 \times \mathrm{C}$ & $30.19 \times \mathrm{C}$ & $\mathrm{C}$ \\
\hline Total Claims & $11.10 \times \mathrm{K}$ & $6.99 \times \mathrm{K}$ & $1.57 \times \mathrm{K}$ & $17.67 \times \mathrm{K}$ & $\mathrm{K}$ \\
\hline Total Field Failures & 1026 & 1084 & 776 & 1228 & 606 \\
\hline
\end{tabular}

The rest of the paper is organized as follows. In Section 2 we describe the components of the dataset being used. Extraction of relevant features is described in Section 3. Section 4 describes the single-vehicle framework for the proposed satisfaction model built on these extracted features. Subsection 4.3 presents the bi-objective optimization problem for obtaining the satisfaction model for a given vehicle model and subsection 4.4 presents the evolutionary optimization method used for solving this problem. Section 5 presents its extension to obtain a generalized satisfaction model when multiple vehicle models are involved. The results and their validation are presented in Section 6. A sensitivity analysis is also performed on the obtained CSI function. Next, Section 7 presents two applications of the quantitative CSI model, which are very relevant from the CRM point of view. Section 8 concludes the paper.

\section{Vehicle Sales and Service Data}

As discussed above, warranty data consists of claims data and supplementary data. The datasets used in this study also contain service repairs made beyond the warranty period and hence we are referring to claims data 
as service data. Supplementary data does not change with time. In our case, the sales data serves as this component.

Before going any further, it would be beneficial to know what attributes of vehicles contribute to satisfaction. A study by J.D. Power and Associates lists the following as critical elements of customer satisfaction [17]:

1. Quality and reliability (24\%) with respect to problems experienced with the vehicle across different vehicle sub-systems.

2. Vehicle appeal (37\%) which includes design, comfort, features, etc.

3. Ownership costs $(22 \%)$ which includes fuel consumption, insurance and costs of post-warranty service or repair.

4. Dealer service satisfaction $(17 \%)$ with respect to service quality, service time, etc.

It can be seen that, satisfaction related to quality, reliability and service contributes close to $50 \%$ towards the overall satisfaction (including some contribution from the ownership costs). In this paper, we are primarily focused on assessing quality, reliability and service satisfaction as they can be quantified through the field failure information obtained from sales and service data. Table 2 shows typical fields found in the sales and service data of a vehicle that can be used to assess this satisfaction.

Table 2: Data fields in the sales and claims data of a vehicle and the notation used in this work.

\begin{tabular}{|l|l|l|l|}
\hline \multicolumn{2}{|c|}{$\begin{array}{l}\text { Vehicle Sales Data } \\
\text { (One-time entry) }\end{array}$} & \multicolumn{2}{c|}{$\begin{array}{l}\text { Vehicle Service Data } \\
\text { (Forth claim of a single visit) }\end{array}$} \\
\hline Vehicle ID No. & VIN & Vehicle ID No. & VIN \\
Sale date & $d_{0}$ & Repair Start date & $d_{i}$ \\
Mileage at sale & $m_{0}$ & Repair End date & $e_{i}$ \\
& & Mileage at repair & $m_{i}$ \\
& & Repair cost & $c_{i}$ \\
& Repair code & $r_{i}$ \\
\hline
\end{tabular}

It is not clear how the data fields in Table 2 can be used directly. Therefore, we first extract certain characteristic features $\mathbf{x}$ from the combined sales and service data, so that the CSI for a particular customer/vehicle can be approximated by a mathematical function $f$,

$$
C S I_{\text {vehicle }}=f(\mathbf{x}) \text {. }
$$

We call $C S I_{\text {vehicle }}$ the CSI model for predicting the customer satisfaction of a given vehicle model. 
From the manufacturer's point of view, a CSI model $(f)$ which can aggregate the views of all the customers in a deterministic way is beneficial for identifying vehicle models with high perceived quality and, more importantly, for highlighting the problem areas across different vehicle models. Taking cues from literature, we can impose certain conditions on the function $f$ so that it achieves the above stated goal. It has been hypothesized that the frequency distribution of customer satisfaction is a theoretically continuous one that is typically skewed to the left [18]. Moreover, it is also widely believed that this distribution is often the convergence of (two or) three nearly normal distributions of (two or) three statistically differing populations - dissatisfied, satisfied and extremely satisfied customers [18]. The work presented in this paper is based on this hypothesis. The direct implication is that, given a vehicle model, most of the customers will have a similar overall perception (satisfaction) of the vehicle model. This results in a $C S I_{\text {vehicle }}$ distribution with low variance as shown in Figure 1. A function $f$ which models the CSIs based on this assumption would also make more sense for averaging over all customers thus obtaining an overall satisfaction value for the vehicle model under consideration. This is what we mean by the bottom-up approach mentioned earlier. By aggregating the CSI vehicle values for all customers corresponding to a vehicle model, a representative CSI value for that vehicle model can be obtained.

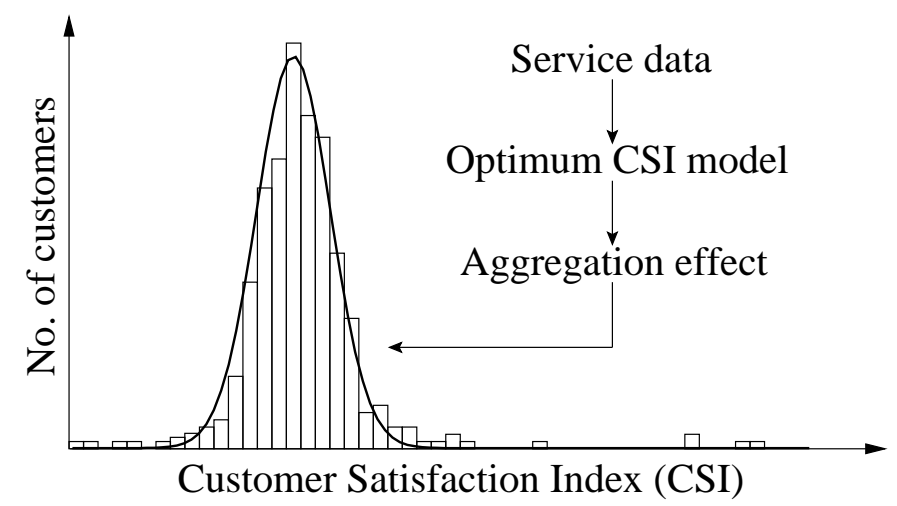

Figure 1: Expected frequency distribution of CSI from the proposed method which makes averaging possible. 


\section{Feature Extraction}

The fields presented in Table 2, though representative of customer satisfaction, cannot directly be used in a mathematical function. To have a computationally tractable method for modeling the CSI, we have identified six features that can be deterministically calculated for each vehicle using the combined sales and service data. More features can be considered to make the study more elaborate. The relation between these features and the satisfaction can also be logically established as shown below:

1. $x_{1}$ : Number of visits made by a customer. The VIN or Vehicle Identification Number is unique for each vehicle. By counting the number of times a particular VIN occurs in the service dataset, the number of visits made by the customer owning that vehicle can be determined. More visits mean lower customer satisfaction and hence the dependency can be modeled as $C S I_{\text {vehicle }} \propto 1 / x_{1}$.

2. $x_{2}$ : Total number of days for which the vehicle was unavailable to the customer. The waiting time for a customer is the calendar difference between the repair start date and the delivery date. We sum these differences over the number of visits to get $x_{2}=\sum_{i=1}^{x_{1}}\left(e_{i}-d_{i}\right)$. Longer waiting times have a negative impact on satisfaction which can be modeled as $C S I_{\text {vehicle }} \propto 1 / x_{2}$.

3. $x_{3}$ : Sum of all service/repair costs. These costs include the labor costs, part costs and miscellaneous costs, if any. The total expenditure on a vehicle for the given period can be obtained as $x_{3}=\sum_{i=1}^{x_{1}} c_{i}$. And logically it follows that $C S I_{\text {vehicle }} \propto 1 / x_{3}$.

4. $x_{4}$ : Average time interval between visits. The time to first visit is the calendar difference between the earliest visit date in the service data and the vehicle sale date. Thereafter, the time interval between subsequent visits can be obtained from the service data alone. The cumulative time intervals are averaged over the number of visits. Mathematically,

$$
x_{4}=\frac{1}{x_{1}}\left(d_{1}-d_{0}+\sum_{i=2}^{x_{1}}\left(d_{i}-d_{i-1}\right)\right) .
$$

A larger value of $x_{4}$ means longer problem-free vehicle use and hence higher customer satisfaction. Therefore, we have $C S I_{\text {vehicle }} \propto x_{4}$.

5. $x_{5}$ : Average miles run between visits. Like the time intervals, the miles run by the vehicle without problems can be obtained from the 
odometer readings in the sales and service data as,

$$
x_{5}=\frac{1}{x_{1}}\left(m_{1}-m_{0}+\sum_{i=2}^{x_{1}}\left(m_{i}-m_{i-1}\right)\right) .
$$

It is easy to conclude that $C S I_{\text {vehicle }} \propto x_{5}$.

6. $x_{6}$ : Sum of problem severity ratings. Each vehicle visit is associated with a repair code $r_{i}$ which defines the type of failure. All repair codes are assigned a severity rating between 1 (minor problem; e.g. oil change) and 5 (major problem; e.g. engine replacement) by domain

experts. Since severity rating has a negative impact on the CSI, we have $C S I_{\text {vehicle }} \propto 1 / x_{6}$.

Notice that the features $x_{4}$ and $x_{5}$ are averages whereas the rest of the features are simply summations. From the OEM's point of view, the cost of overestimating the CSI is usually more than the cost of underestimating it. Since both the sum of days and the sum of miles between visits positively affect the CSI, averaging them over the number of visits reduces the chance of overestimating the customer satisfaction for any particular customer.

\section{Single Vehicle CSI Model}

The characteristic features extracted above are still not directly usable since they vary in different ranges. A pre-processing step is needed for this purpose. In this section, we discuss the procedure for obtaining an optimized CSI function for each vehicle model one at a time, then in the next section, we shall discuss a way to come up with a multiple vehicle CSI function.

\subsection{Pre-processing of Warranty Data}

Table 3 shows some statistics of the features extracted from the service data of the five vehicle models that we use in this work. It is apparent that the direct use of these features for constructing $f$ will lead to a biased CSI model, since numerically they will have varying effects on the CSI. Therefore, a normalization procedure is adopted to map the features to the range $[0,1]$ using,

$$
x_{i}^{n r}=\frac{x_{i}-\min _{j} x_{i}^{(j)}}{\max _{j} x_{i}^{(j)}-\min _{j} x_{i}^{(j)}} \quad \forall i \in\{1,2, \ldots, 6\}, j \in\{1,2, \ldots, C\},
$$

where $C$ is the total number of customers of a vehicle model. It is important 
Table 3: Some statistics of extracted features for all vehicle models.

\begin{tabular}{|c|c|c|c|c|c|c|c|}
\hline & Stat. & $x_{1}$ & $x_{2}$ & $x_{3}$ & $x_{4}$ & $x_{5}$ & $x_{6}$ \\
\hline \multirow{3}{*}{\begin{tabular}{l}
-1 \\
$\overrightarrow{0}$ \\
$\frac{0}{0}$ \\
\multirow{2}{*}{}
\end{tabular}} & Min. & 1 & 1 & 0 & 1 & 1 & 1 \\
\hline & Max. & 20 & 357 & 11253.76 & 429 & 799686 & 50 \\
\hline & Mean & 1.39 & 1.46 & 155.93 & 110.63 & 5317.23 & 3.14 \\
\hline \multirow{3}{*}{$\begin{array}{l}N \\
\stackrel{N}{0} \\
\mathbb{0} \\
\Sigma\end{array}$} & Min. & 1 & 1 & 0 & 1 & 1 & 1 \\
\hline & Max. & 12 & 371 & 8195.94 & 395 & 115671 & 39 \\
\hline & Mean & 1.32 & 1.37 & 199.07 & 137 & 7810.22 & 3.09 \\
\hline \multirow{3}{*}{$\begin{array}{l}\infty \\
0 \\
0 \\
0 \\
\Sigma\end{array}$} & Min. & 1 & 1 & 1.91 & 1 & 1 & 1 \\
\hline & Max. & 10 & 77 & 12188.07 & 408 & 999959 & 51 \\
\hline & Mean & 1.67 & 1.74 & 349.01 & 114.57 & 5502.76 & 4.21 \\
\hline \multirow{3}{*}{$\begin{array}{l}\vec{H} \\
0 \\
0 \\
0 \\
\dot{0}\end{array}$} & Min. & 1 & 1 & 0 & 1 & 1 & 1 \\
\hline & Max. & 17 & 368 & 9985.60 & 433 & 333024 & 45 \\
\hline & Mean & 1.45 & 1.59 & 148.10 & 129.33 & 6573.53 & 2.65 \\
\hline \multirow{3}{*}{$\begin{array}{l}10 \\
0 \\
0 \\
0 \\
0\end{array}$} & Min. & 1 & 1 & 5 & 1 & 1 & 1 \\
\hline & Max. & 10 & 248 & 9134 & 459 & 78501 & 35 \\
\hline & Mean & 2.01 & 2.35 & 458.71 & 118.70 & 5162.20 & 5.03 \\
\hline
\end{tabular}

to realize that such a linear normalization does not affect the frequency distribution.

Second, keeping in mind different logical dependencies established earlier, we now introduce the following feature transformation for simplicity of notation, so that an increase in the value of $X_{i}$ leads to a better CSI,

$$
\begin{array}{ll}
X_{i}=\frac{1}{\left(1+x_{i}^{n r}\right)} & , \text { for } i \in\{1,2,3,6\}, \\
X_{i}=\left(1+x_{i}^{n r}\right) & , \text { for } i \in\{4,5\} .
\end{array}
$$

This transformation allows us to use a monotonic CSI function as a variation of each $X_{i}$. In this work, we call $X_{i}$ 's as the transformed features in contrast to the $x_{i}$ 's introduced in the last section, which are called extracted features. The addition of the constant (one here) in Equation (5) and (6) ensures that the CSI does not approach infinity for vehicles with minimum corresponding $x_{i}$. 


\subsection{Mathematical Structure of CSI Function}

To arrive at a mathematical form for the CSI function, we now define a functional form:

$$
C S I_{\text {vehicle }}=f\left(X_{1}, X_{2}, X_{3}, X_{4}, X_{5}, X_{6}\right) \text {. }
$$

The function $f$ is not known a priori, except to know that it is a monotonic function of the six transformed features. However, there are certain expectations we have for the CSI function. As stated earlier, our primary goal is to obtain a CSI model which will have a low variance (as shown in Figure 1) for a given vehicle model for the given data. Secondly, the model should be flexible enough to differentiate between two or more vehicle models which have clearly different perceived quality in the market. This is our secondary goal. We use a parametric mathematical model which allows the features to interact freely with each other. The parameters of the model are obtained using an algorithm which satisfies both the goals. Our assumed model is composed of six terms, $T_{l}$. Each term is a product of different transformed features given by,

$$
T_{l}=\prod_{i=1}^{6} X_{i}^{\alpha_{i l} \beta_{i l}} \quad \forall i, l \in\{1, \ldots, 6\} .
$$

Here $\beta_{i l}$ 's are Boolean decision variables which decide the presence (when $\beta_{i l}=1$ ) or absence (when $\beta_{i l}=0$ ) of the $i$-th transformed feature in the $l$-th term. When $\beta_{i l}=1, \alpha_{i l}$ denotes the corresponding exponent.

The six multiplicative terms can in turn be multiplied or added to each other. Again, a Boolean encoding decides which terms to multiply and which ones to add. We adopt 1 for ' + ' operator and 0 for ' $x$ ' operator. These intermediate Boolean variables are represented by $\gamma_{l}$. The inverse operations (division and subtraction) are not considered since the dependency of $C S I_{\text {vehicle }}$ to each extracted feature $x_{i}$ is already incorporated into the transformed features $X_{i}$ 's. For the same reason the powers $\alpha_{i l}$ 's are considered to be non-negative real numbers lying between 0 and 1 . With six terms, we have five additional Boolean decision variables. The 41 (=36 $\beta$ 's $+5 \gamma^{\prime}$ s) Boolean variables can be represented using a binary string. In addition, we have 36 real-variables ( $\alpha$ 's).

Figure 2 shows an example of the representation scheme adopted in this paper. Every seventh bit is the variable $\gamma_{l}$ which defines the arithmetic operation between the $T_{l}$-th and $T_{l+1}$-th terms. The usual order of precedence is followed for the operators (multiplication and then addition) to evaluate 


\begin{tabular}{|c|c|c|c|c|c|c|c|c|c|c|c|c|c|c|c|c|c|c|c|c|c}
$\beta_{11}$ & $\beta_{21}$ & $\beta_{31}$ & $\beta_{41}$ & $\beta_{51}$ & $\beta_{61}$ & $\gamma_{1}$ & $\beta_{12}$ & $\beta_{22}$ & $\beta_{32}$ & $\beta_{42}$ & $\beta_{52}$ & $\beta_{62}$ & $\gamma_{2}$ & $\beta_{13}$ & $\beta_{23}$ & $\beta_{33}$ & $\beta_{43}$ & $\beta_{53}$ & $\beta_{63}$ & $\gamma_{3}$ \\
\hline 0 & 1 & 0 & 0 & 1 & 1 & 1 & 0 & 1 & 0 & 1 & 0 & 1 & 0 & 1 & 1 & 1 & 0 & 0 & 0 & 1 & $\cdots \cdots$ \\
\hline
\end{tabular}

Figure 2: Binary representation for the proposed adaptive form of the CSI model.

the CSI. Whenever a term evaluates to unity (i.e. when $\left.\beta_{i l}=0 \forall i\right)^{1}$, it is simply ignored in further computations. The illustrated string evaluates to

$$
\begin{aligned}
C S I_{\text {vehicle }} & =T_{1}+T_{2} \times T_{3}+\cdots \\
& =X_{2}^{\alpha_{21}} X_{5}^{\alpha_{51}} X_{6}^{\alpha_{61}}+X_{2}^{\alpha_{22}} X_{4}^{\alpha_{42}} X_{6}^{\alpha_{62}} \times X_{1}^{\alpha_{13}} X_{2}^{\alpha_{23}} X_{3}^{\alpha_{33}}+\cdots \\
& =X_{2}^{\alpha_{21}} X_{5}^{\alpha_{51}} X_{6}^{\alpha_{61}}+X_{1}^{\alpha_{13}} X_{2}^{\alpha_{22}+\alpha_{23}} X_{3}^{\alpha_{33}} X_{4}^{\alpha_{42}} X_{6}^{\alpha_{62}}+\cdots
\end{aligned}
$$

This modeling approach resembles a genetic programming module [19] with $\mathcal{T}=\left\{X_{i} \forall i\right\}$ as the terminal set and $\mathcal{F}=\{\times,+\}$ as the functional set without introducing the computational cost associated with such generic systems.

\subsection{Formulating an Optimization Problem}

For achieving a customer level CSI model we now focus on our primary goal of obtaining an optimized CSI function which, when evaluated for different customers produces a distribution with low variance. A narrower CSI distribution means better agreement among the customers thus making averaging more sensible for obtaining the overall satisfaction. It is intuitive that this can be accomplished by minimizing the variance (or standard deviation) of the CSI values of all customers. However, there are two situations which can lead to trivial solutions.

Firstly, since the exponents $\alpha_{i l}$ 's are non-negative, a simple minimization of the variance causes them to approach the lower bound of zero giving rise to severely right skewed CSI distributions after normalization. This is shown in Figure 4, described later. To avoid this, we incorporate a second 'helper objective' which minimizes the skewness of the distribution. Secondly, the presence of Boolean variables may cause $\beta_{i l}=0 \forall i, l$ giving a trivial distribution of zero variance. The following set of constraints are used to prevent this situation.

$$
\sum_{l} \beta_{i l} \geq 1 \quad \forall i \in\{1, \ldots, 6\}
$$

\footnotetext{
${ }^{1} \alpha_{i l}=0 \forall i$ is highly improbable.
} 
which basically impose that each extracted feature $X_{i}$ is used in at least one of the terms. This also makes practical sense since the six extracted features are the only information that can be derived from the sales and service data and we would want the resulting CSI model to use all of them.

This optimization is carried out using a genetic algorithm (GA). The binary representation of the model discussed above was designed with this in mind. Equation (9) gives the bi-objective optimization problem formulation for finding the customer level CSI model from the sales and service data of $C$ customers of a vehicle model.

$$
\begin{aligned}
& \text { Minimize } \sigma \\
& \text { Minimize } \quad|g| \\
& \text { Subject to } \quad \sum_{l} \beta_{i l} \geq 1 \quad \forall \quad i \in\{1, \ldots, 6\} \text {, } \\
& 36 \text { real variables: } \quad 0 \leq \alpha_{i l} \leq 1 \quad \forall \quad i, l \in\{1, \ldots, 6\} \text {, } \\
& 36 \text { Boolean variables: } \quad \beta_{i l} \in\{0,1\} \quad \forall \quad i, l \in\{1, \ldots, 6\} \text {, } \\
& \text { 5 Boolean variables: } \quad \gamma_{l} \in\{0,1\} \quad \forall \quad l \in\{1, \ldots, 5\} \text {, } \\
& \text { where, } \\
& \begin{aligned}
\sigma & =\sqrt{\frac{1}{C} \sum_{j=1}^{C}\left(C S I_{\text {vehicle }, j}^{n r}-\mu\right)^{2}}, \quad \mu=\sum_{j=1}^{C} C S I_{\text {vehicle }, j}^{n r} / C, \\
g & =\frac{1}{C} \sum_{j=1}^{C}\left(C S I_{\text {vehicle }, j}^{n r}-\mu\right)^{3} / \sigma^{3} .
\end{aligned}
\end{aligned}
$$

where $C S I_{\text {vehicle, } j}^{n r}$ is the normalized value of $C S I_{\text {vehicle }}$ evaluated for the $j$ th customer. The normalization is linear between 0 and 1 , performed using

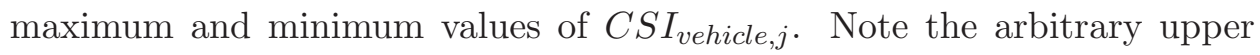
bound on $\alpha_{i l}$ 's. Any other value may also be used.

\subsection{Applying Evolutionary Optimization Method}

Due to the bi-objective nature of the above optimization problem and due to non-linearities and non-differentiability in the definitions of the objective functions, we choose an evolutionary multi-objective optimization (EMO) method - the elitist non-dominated sorting GA or NSGA-II [20].

Figure 3 shows the non-dominated solutions obtained by solving (9) for each of the five vehicle models (refer Table 1) individually using the NSGAII procedure. All fronts are obtained using the following standard parameter settings:

1. Population size: 2,000, 


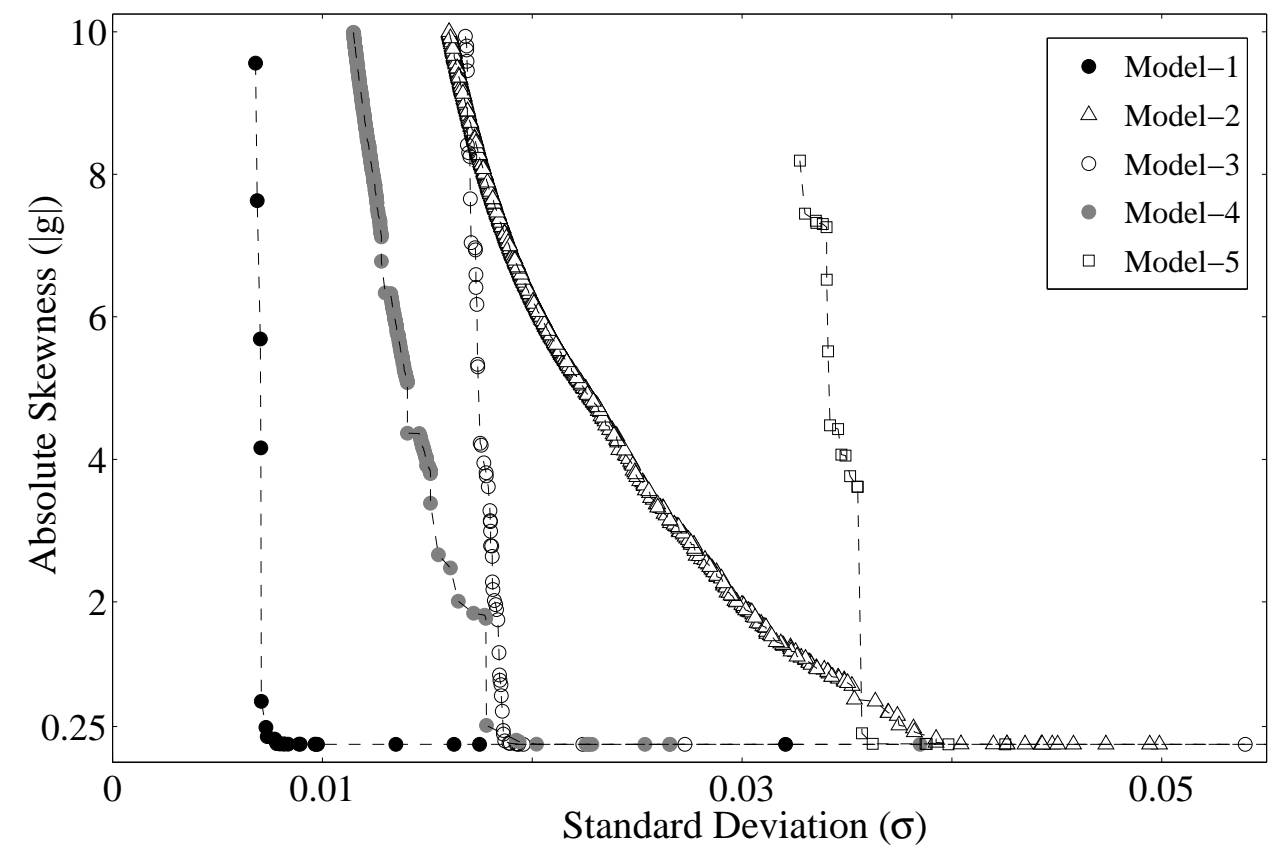

Figure 3: Non-dominated solution sets for the five vehicle models considered in this study, obtained by solving Equation (9). Note that each point shown on these trade-off fronts represents a different CSI model.

2. Number of generations: 500,

3. Tournament selection with size 2 ,

4. Simulated binary crossover [21] with $p_{c}=0.9$ and $\eta_{c}=10$ for $\alpha$ 's,

5. Polynomial mutation [22] $p_{m}=0.05$ and $\eta_{m}=50$ for $\alpha$ 's,

6. Single-point crossover with $p_{c}=0.9$ for binary string ( $\beta$ 's and $\gamma$ 's),

7. Bitwise mutation with $p_{m}=0.15$ for binary string ( $\beta$ 's and $\gamma$ 's).

The population size and number of generations are set by observing the number of function evaluations (per objective) after which no further improvement in the non-dominated front occurs. Multiple runs have indicated this value to be $10^{6}$ function evaluations. We chose a relatively high population size of 2,000 (coupled with 500 generations to bring function evaluations to $10^{6}$ ) and a high crossover probability of 0.9 to promote exploration. No experimentation was performed with respect to mutation probabilities $\left(p_{m}\right)$ and distribution indices $\left(\eta_{c}\right.$ and $\left.\eta_{m}\right)$ [21].

Each trade-off solution in Figure 3 represents a different CSI model. The sharp kink in each of the trade-off fronts indicates the presence of a 


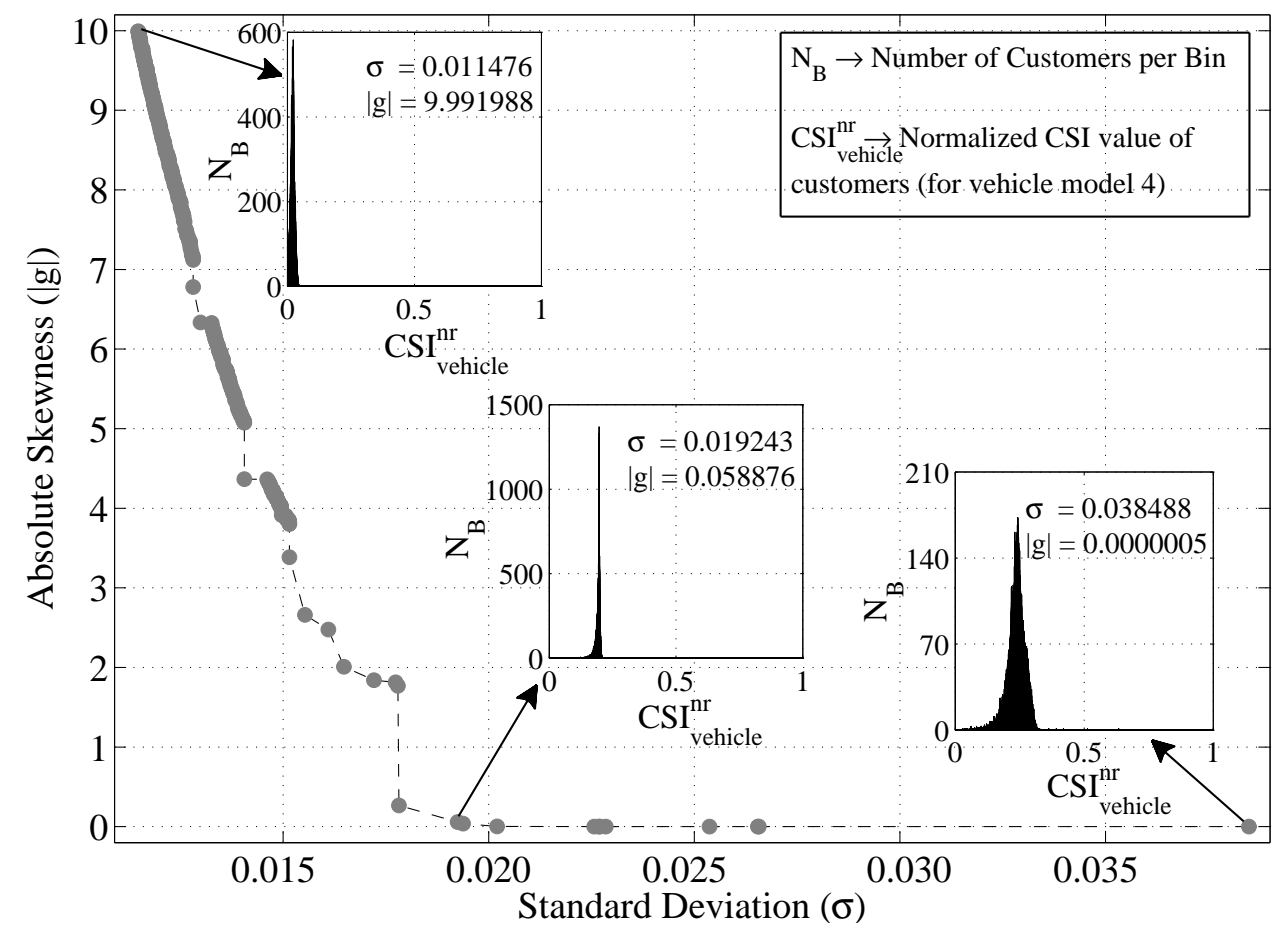

Figure 4: The figure shows the non-dominated solution set obtained from solving Equation (9) for Model 4. The three insets show the CSI distributions corresponding to, (a) the leftmost point of the trade-off front, (b) a point in the knee region of the trade-off front, and, (c) the rightmost point of the trade-off front.

knee [23]. The knee point of a two-dimensional Pareto-optimal front is the solution which gives the best trade-off with respect to both objectives. Due to this characteristic, it is often the preferred solution. Figure 4 shows the CSI distributions corresponding to three different points on the tradeoff front of Model 4. Note that the leftmost solution is a trivial solution obtained when all $\alpha$ 's approach their lower bounds as stated earlier. On the other hand, the rightmost solution yields a relatively wide CSI distribution which makes it difficult to estimate the overall CSI rating of the vehicle model from the CSI values of individual customers, because an averaged CSI value in such cases is not representative of the entire data and hence is not meaningful. The second (helper) objective allows many such CSI models to coexist in the NSGA-II population, among which the knee solution is found to be the most favorable one. Other solutions near the knee region 
Table 4: Objective function values and the knee-point CSI models for all vehicle models.

\begin{tabular}{|c|c|c|}
\hline & & Knee-point solution details \\
\hline \multirow[b]{2}{*}{$\begin{array}{l}-1 \\
\frac{0}{0} \\
\frac{0}{2} \\
\sum\end{array}$} & Objectives & $\sigma=0.0071,|g|=0.0021$ \\
\hline & $C S I_{\text {vehicle }}$ & $\begin{array}{c}T_{1}+T_{2} \times T_{3}+T_{4}+T_{5}+T_{6} \\
=X_{2}^{0.0632} X_{3}^{0.0184} X_{5}^{0.0696}+X_{2}^{0.0632} X_{5}^{0.9175}+X_{2}^{0.5889} X_{3}^{0.0704} \\
+X_{1}^{0.0153} X_{2}^{0.7322} X_{6}^{0.0375}+X_{2}^{0.9479} X_{4}^{0.0011}\end{array}$ \\
\hline \multirow[b]{2}{*}{$\begin{array}{l}N \\
\frac{0}{0} \\
\stackrel{0}{0} \\
\sum\end{array}$} & Obje & $\sigma=0.0392,|g|=0.0253$ \\
\hline & $C S I_{\text {vehicle }}$ & $\begin{array}{c}T_{1}+T_{2} \times T_{3} \times T_{4} \times T_{5}+T_{6} \\
=X_{3}^{0.9238} X_{4}^{0.0092} X_{5}^{0.0048}+X_{1}^{0.3450} X_{2}^{0.8507} X_{3}^{0.9177} X_{4}^{0.0071} \\
+X_{2}^{0.0292} X_{3}^{0.2830} X_{5}^{0.7601}+X_{2}^{0.1085} X_{3}^{0.8603}\end{array}$ \\
\hline \multirow{2}{*}{ 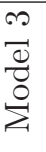 } & Obje & $\sigma=0.0178,|g|=0.2665$ \\
\hline & $C S I_{\text {vehicle }}$ & $\begin{array}{r}T_{1}+T_{2} \times T_{3} \times T_{4} \times T_{5}+T_{6} \\
=X_{1}^{0.1089} X_{2}^{0.2060} X_{3}^{0.9139}+X_{2}^{0.4329} X_{3}^{0.9731}\end{array}$ \\
\hline \multirow[b]{2}{*}{ 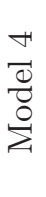 } & Objectives & $\sigma=0.0187,|g|=0.0498$ \\
\hline & $C S I_{\text {vehicle }}$ & $\begin{array}{c}T_{1}+T_{2} \times T_{3}+T_{4}+T_{5}+T_{6} \\
=X_{2}^{0.8242} X_{6}^{0.0259}+X_{1}^{0.0030} X_{2}^{0.9037} X_{3}^{0.0217}+X_{5}^{0.7632} \\
+X_{2}^{0.2979}+X_{3}^{0.0283}\end{array}$ \\
\hline \multirow[b]{2}{*}{ 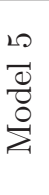 } & Objectives & $\sigma=0.0362,|g|=0.0072$ \\
\hline & $C S I_{\text {vehicle }}$ & $\begin{array}{c}T_{1}+T_{2} \times T_{3}+T_{4}+T_{5}+T_{6} \\
=X_{2}^{0.7563} X_{4}^{0.0287} X_{5}^{0.0230} X_{6}^{0.0303}+X_{2}^{0.4977} X_{5}^{0.6763}+X_{2}^{0.8656} \\
+X_{2}^{0.9701} X_{6}^{0.0142}+X_{2}^{0.8672} X_{3}^{0.0151}\end{array}$ \\
\hline
\end{tabular}

also yield similar distributions, making this region a potential space for obtaining good CSI models. We use the bend-angle approach proposed in [23] to identify the knee solution. Table 4 shows the knee-point CSI models and their corresponding objective values for all five vehicle models obtained from the trade-off fronts shown in Figure 3. Note that for all five models, both $\sigma$ and $|g|$ values are small.

\section{Multiple Vehicle CSI Model}

The customer level CSI modeling technique presented above fulfill our primary goals of obtaining a low variance and low skewness CSI distribution. However, as seen in Table 4 the obtained CSI models differ between vehicle models and hence do not provide a common basis for comparing or ranking them. Our secondary goal, as stated earlier, is therefore to modify the prob- 
lem formulation in (9) so that a single CSI model can be used to differentiate between two or more vehicle models as distinctly as possible. For this purpose, we propose to maximize the absolute difference between the average CSI values of different combinations of vehicle models under consideration. Since this can be time-consuming, we first reduce the computational effort for obtaining a specific CSI function for each vehicle model.

The results from customer level CSI models tell us that the best CSI models are obtained in the knee region. We can therefore constrain the search space to the knee regions of all five vehicle models by converting the variance and skewness objectives into additional constraints. This will then allow us to employ a single-objective optimization method. From Figure 3, it is clear that the knee regions of all the five efficient fronts corresponding to the customer level CSI models of the five vehicle models are bounded by $\sigma \leq 0.05$ and $|g| \leq 0.25$. The single-objective optimization problem proposed for multiple vehicle CSI modeling can now be stated as:

\begin{tabular}{|c|c|c|c|}
\hline Maximize & $\sum_{\{m, n \mid m \neq n\}} \mid \mu$ & 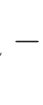 & \\
\hline Subject to & $\sigma_{m} \leq 0.05$ & $\forall$ & $m \in\{1,2, \ldots, 5\}$ \\
\hline & $\left|g_{m}\right| \leq 0.25$ & $\forall$ & $m \in\{1,2, \ldots, 5\}$ \\
\hline & $\sum_{l} \beta_{i l} \geq 1$ & $\forall$ & $i \in\{1,2, \ldots, 6\}$ \\
\hline where, & & & \\
\hline 36 real variables: & $0 \leq \alpha_{i l} \leq 1$ & $\forall$ & $i, l \in\{1,2, \ldots, 6\}$, \\
\hline 36 Boolean variables: & $\beta_{i l} \in\{0,1\}$ & $\forall$ & $i, l \in\{1,2, \ldots, 6\}$, \\
\hline 5 Boolean variables: & $\gamma_{l} \in\{0,1\}$ & $\forall$ & $l \in\{1,2, \ldots, 5\}$. \\
\hline
\end{tabular}

Here, $m$ and $n$ represent the indices of the vehicle models considered for obtaining the multiple vehicle CSI model. $\sigma_{m}, \mu_{m}$ and $g_{m}$ are respectively the standard deviation, mean and skewness of the normalized CSI values of the $m$-th vehicle model (having $C_{m}$ customers). For the five vehicle models, we can use all or any combinations of them to obtain a corresponding multiple vehicle CSI model. Again, an evolutionary optimization algorithm (EA) is used to solve the above problem. The sales and service data of all the vehicle models considered are given as input.

In the following discussion we refer to any combination of vehicle models as a Training Set (TS). For simplicity of notation, we represent a training set as TS\{List of vehicle models $\}$ and the corresponding CSI model as, $C S I_{\{\text {List of vehicle models }\}}$. For example, the training set containing Models 2,3 and 4 is TS $\{2,3,4\}$ and its corresponding CSI model will be referred to as $C S I_{\{2,3,4\}}$. 


\section{Results and Discussion}

Due to the large number of customers involved with each vehicle model and the use of a population based method, all optimization runs are computationally expensive. For example, solving Equation (9) for Model 4 on a 3GB machine with 4-core processor takes more than 48 hours to complete $10^{6}$ evaluations. The processor burden is even higher when multiple models are considered together to solve Equation (10). Since the evaluation of the CSI for one customer is independent of that for another, there is scope for parallelization without affecting the EA operators. All optimization runs required in this work were performed on an NVIDIA Tesla C1060 Graphics Processing Unit containing 30 symmetric multi-processors using the CUDA architecture. The computation of the CSI function for different customers is assigned to multiple threads operating simultaneously in the device. This reduces the computational time by a factor proportional to the total number of customers.

The five vehicle models in this study have been chosen from different market segments as shown in Table 1. These models have been in the market for some time and survey based assessment is available for them. Unlike the American Customer Satisfaction Index and surveys by J.D. Power and Associates, Consumer Reports surveys assess customer satisfaction based on three aspects: performance, safety and reliability. In the context of the methods proposed in this paper, where we use service data for CSI modeling, Consumer Reports reliability ratings provide the most relevant assessment for comparisons. According to a Consumer Reports study [24], the order of reliability ratings of the five vehicle models is given by,

$$
C S I_{5} \prec C S I_{3} \prec C S I_{1} \prec C S I_{4} \approx C S I_{2} .
$$

meaning that Model 5 has the worst overall customer perception, followed by Model 3 and so on. Consumer satisfaction indices for Models 2 and 4 are similar and they have the best overall reliability rating among all five models.

In this section, we first discuss in detail the results from the multiple vehicle CSI modeling of TS $\{1,2,3\}$ as an illustrative example and compare the CSI ranking against (11). Later we summarize the results for all possible model combinations and choose the best CSI model based on statistical testing.

\subsection{CSI Model for TS\{1,2,3\}: An Illustrative Example}

The GA parameters for solving (10) for TS $\{1,2,3\}$ are mostly the same as those specified for NSGA-II in Section 4.4 except the population size and 
number of generations which are set to 500 and 10000 respectively. Constraints are handled using the penalty-parameter-less approach described in [25]. The optimal CSI model obtained by solving Equation (10) for Models 1,2 , and 3 is

$$
\begin{aligned}
C S I_{\{1,2,3\}}= & T_{1}+T_{2} \times T_{3} \times T_{4}+T_{5}+T_{6} \\
= & X_{1}^{0.5226} X_{5}^{0.7215}+X_{2}^{0.8558} X_{3}^{0.6597} X_{4}^{0.0349} X_{5}^{0.8129} \\
& +X_{1}^{0.7226} X_{5}^{0.5773}+X_{1}^{0.0255} X_{3}^{0.2429} X_{5}^{0.1124}
\end{aligned}
$$

The optimized CSI model $C S I_{\{1,2,3\}}$ is now used to evaluate the CSI values for all the customers in $\operatorname{TS}\{1,2,3\}$. Figure 5 shows the normalized CSI distributions obtained for these vehicle models. The normalization is performed over all the customers in $\operatorname{TS}\{1,2,3\}$. Normal distribution curves

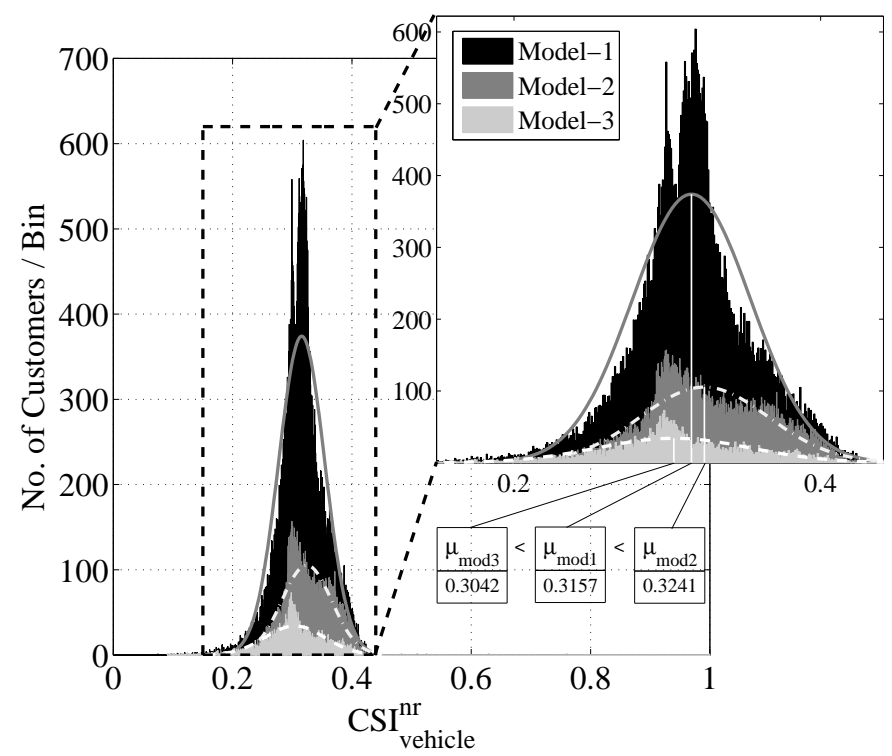

Figure 5: Normalized CSI distributions obtained using $C S I_{\{1,2,3\}}$ for Models 1,2 and 3 . The optimized objective function value is $\left|\mu_{1}-\mu_{2}\right|+\mid \mu_{1}-$ $\mu_{3}|+| \mu_{2}-\mu_{3} \mid=0.039803$.

are fitted to the distributions in Figure 5 to clearly show the location of means. From Figure 5, it can be clearly seen that our primary goal of low variance and low-skewness distribution is met for all three vehicle models. The mean of each distribution can therefore be considered as overall CSI for each vehicle model. The inset in Figure 5 shows the relative positions of the 
means of the three distributions clearly revealing the satisfaction rating in the order,

$$
C S I_{3} \prec C S I_{1} \prec C S I_{2} .
$$

Here, mean of the CSI distribution of Model $v$ has simply been denoted by $C S I_{v}$. The above agrees with the Consumer Reports rating in (11).

\subsection{Statistical Hypothesis Testing}

Table 5 shows some statistical characteristics of the three CSI distributions shown in Figure 5. Numerically, the mean CSI values for the three vehicle models are observed to be very close. This raises concerns regarding the statistical significance of (13). For analyzing this further, let us consider the following null hypothesis,

$$
H_{0}: M_{m}-M_{n}=0
$$

where $M_{m}$ and $M_{n}$ are the population means for Models $m$ and $n$ respectively. The use of Welch's t-test has been suggested in [26] for independent or unpaired (in statistical hypothesis testing terminology) samples with unequal sizes and variances. This test is basically an extension of the Student's two sample $t$-test. The $t$ statistic is modified to accommodate unequal sample sizes as follows:

$$
t=\frac{\mu_{m}-\mu_{n}}{\sqrt{\frac{\sigma_{m}^{2}}{c_{m}}+\frac{\sigma_{n}^{2}}{c_{n}}}} .
$$

The degree of freedom $(\nu)$ expression is calculated using,

$$
\nu=\frac{\left(\frac{\sigma_{m}^{2}}{c_{m}}+\frac{\sigma_{n}^{2}}{c_{n}}\right)^{2}}{\frac{\sigma_{m}^{4}}{c_{m}^{2}\left(c_{m}-1\right)}+\frac{\sigma_{n}^{4}}{c_{n}^{2}\left(c_{n}-1\right)}}
$$

Table 5: Statistical characteristics of the three CSI distributions shown in Figure 5.

\begin{tabular}{|c|c|c|c|}
\hline Model No. & $\begin{array}{c}\text { Mean } \\
\mu_{m}\end{array}$ & $\begin{array}{c}\text { Std. dev. } \\
\sigma_{m}\end{array}$ & $\begin{array}{c}\text { Skewness } \\
\left|g_{m}\right|\end{array}$ \\
\hline Model 1 & 0.315774 & 0.038656 & 0.238562 \\
\hline Model 2 & 0.324148 & 0.041722 & 0.245239 \\
\hline Model 3 & 0.304247 & 0.049920 & 0.207301 \\
\hline
\end{tabular}


Note that $\mu$ and $\sigma$ here are the sample mean and standard deviation respectively. As is customary in such tests, we use $\alpha=5 \%$ significance level. Table 6 shows the results of three Welch $t$-tests performed on all pairs of Models 1, 2 and 3. Since none of the confidence intervals encloses the hy-

Table 6: Welch's t-test statistics.

\begin{tabular}{|c|c|c|c|c|c|}
\hline $\begin{array}{c}\text { Model } \\
\text { Pair } \\
\{\mathrm{m}, \mathrm{n}\}\end{array}$ & $t$ & $\nu$ & $\begin{array}{c}95 \% \text { confidence } \\
\text { interval }\left(M_{m}-M_{n}\right)\end{array}$ & $\mu_{m}-\mu_{n}$ & Implication \\
\hline$\{1,2\}$ & -28.72 & 66080.89 & $(-0.0089,-0.0078)$ & -0.008374 & $C S I_{1} \prec C S I_{2}$ \\
$\{1,3\}$ & 15.35 & 5246.97 & $(0.0101,0.0130)$ & 0.011527 & $C S I_{1} \succ C S I_{3}$ \\
$\{2,3\}$ & 26.00 & 5663.99 & $(0.0184,0.0214)$ & 0.019901 & $C S I_{2} \succ C S I_{3}$ \\
\hline
\end{tabular}

pothesized mean difference value of zero and the difference between sample means for all vehicle model pairs lie within the confidence interval, the null hypothesis $H_{0}$ can be rejected in all three tests with $95 \%$ confidence. The alternate hypothesis stating that the difference between the means is statistically significant for all three model pairs is hence accepted in all cases. The implication of each test is summarized in the last column of Table 6 .

The disadvantage of using multiple pairwise comparisons as shown above is that the probability of making at least one Type I error (incorrect rejection of true null hypothesis) increases with the number of comparisons $N C$ as $1-(1-\alpha)^{N C}$. For $T S\{1,2,3,4,5\}$ where we require 10 comparisons, this probability comes out to be greater than $40 \%$. The Tukey-Kramer multiple comparison test [27] is used instead when there are more than two groups to be compared and their sample sizes are unequal. In a single step, it shows all pairs that have significantly different means. In this paper, we implement the Tukey-Kramer test using Matlab's multcompare function. Detailed results are shown later in Table 7 .

\subsection{CSI Models for Remaining Training Sets}

In this section, we solve (10) for all training sets. Additionally, regardless of the number of vehicle models used, we apply the obtained CSI model in each case to all five vehicle models to test the generality of the obtained function $^{2}$. Statistical tests are also performed in all cases. The procedure for obtaining the CSI precedence order and statistical testing remains exactly the same as in the previous section.

\footnotetext{
${ }^{2}$ This is the reason for calling vehicle model combinations as training sets.
} 
Table 7: Overall CSI precedence relations for all five vehicle models obtained from all possible vehicle model combinations. CSI models which are infeasible or which resulted in incorrect CSI precedence relations (against (11)) are marked in gray. The best CSI model is shown in bold.

\begin{tabular}{|c|c|c|c|c|c|c|}
\hline \multirow{2}{*}{\multicolumn{2}{|c|}{$\begin{array}{c}\text { Model } \\
\text { Training Sets }\end{array}$}} & \multirow{2}{*}{$\begin{array}{c}\text { Predicted } \\
\text { CSI precedence } \\
\text { (Training Sets }+ \text { Test Sets) }\end{array}$} & \multicolumn{2}{|c|}{$\begin{array}{l}\text { Objective Value } \\
\quad\left(\times 10^{-2}\right)\end{array}$} & \multicolumn{2}{|c|}{$\begin{array}{l}\text { Statistically } \\
\text { Significant? }\end{array}$} \\
\hline & & & Actual & Scaled & Welch & Tukey-Kramer \\
\hline \multirow{10}{*}{ II-TS } & $\{1,2\}$ & $C S I_{5} \prec C S I_{3} \prec C S I_{1} \prec C S I_{4} \prec C S I_{2}$ & 1.3847 & 1.3847 & YES & YES \\
\hline & $\{1,3\}$ & $C S I_{5} \prec C S I_{3} \prec C S I_{1} \prec C S I_{2} \prec C S I_{4}$ & 1.3820 & 1.3820 & YES & YES \\
\hline & $\{1,4\}$ & $C S I_{5} \prec C S I_{3} \prec C S I_{1} \prec C S I_{2} \prec C S I_{4}$ & 1.3770 & 1.3770 & YES & YES \\
\hline & $\{1,5\}$ & $C S I_{5} \prec C S I_{3} \prec C S I_{1} \prec C S I_{2} \prec C S I_{4}$ & 3.2239 & 3.2239 & YES & YES \\
\hline & $\{2,3\}$ & $C S I_{5} \prec C S I_{3} \prec C S I_{1} \prec C S I_{4} \prec C S I_{2}$ & 2.0196 & 2.0196 & NO & NO \\
\hline & $\{2,4\}$ & $C S I_{5} \prec C S I_{3} \prec C S I_{1} \prec C S I_{4} \prec C S I_{2}$ & 0.9831 & 0.9831 & YES & YES \\
\hline & $\{2,5\}$ & $C S I_{5} \prec C S I_{3} \prec C S I_{2} \prec C S I_{1} \prec C S I_{4}$ & NA & NA & YES & NO \\
\hline & $\{3,4\}$ & $C S I_{5} \prec C S I_{3} \prec C S I_{1} \prec C S I_{2} \prec C S I_{4}$ & 2.3567 & 2.3567 & YES & YES \\
\hline & $\{3,5\}$ & No Feasible Solution Found & NA & NA & NA & NA \\
\hline & $\{4,5\}$ & $C S I_{5} \prec C S I_{3} \prec C S I_{1} \prec C S I_{2} \prec C S I_{4}$ & 4.2274 & 4.2274 & YES & YES \\
\hline \multirow{10}{*}{ III-TS } & $\{1,2,3\}$ & $C S I_{5} \prec C S I_{3} \prec C S I_{1} \prec C S I_{4} \prec C S I_{2}$ & 3.9803 & 1.3268 & YES & YES \\
\hline & $\{1,2,4\}$ & $C S I_{5} \prec C S I_{3} \prec C S I_{1} \prec C S I_{4} \prec C S I_{2}$ & 2.6595 & 0.8865 & YES & YES \\
\hline & $\{1,2,5\}$ & $C S I_{5} \prec C S I_{3} \prec C S I_{1} \prec C S I_{2} \prec C S I_{4}$ & 6.5799 & 2.1933 & YES & YES \\
\hline & $\{1,3,4\}$ & $C S I_{5} \prec C S I_{3} \prec C S I_{1} \prec C S I_{2} \prec C S I_{4}$ & 4.6537 & 1.5512 & YES & YES \\
\hline & $\{1,3,5\}$ & $C S I_{5} \prec C S I_{3} \prec C S I_{1} \prec C S I_{4} \prec C S I_{2}$ & 3.6521 & 1.2174 & YES & YES \\
\hline & $\{1,4,5\}$ & $C S I_{5} \prec C S I_{3} \prec C S I_{1} \prec C S I_{2} \prec C S I_{4}$ & 8.0151 & 2.6717 & YES & YES \\
\hline & $\{2,3,4\}$ & $C S I_{5} \prec C S I_{3} \prec C S I_{1} \prec C S I_{2} \prec C S I_{4}$ & 4.7065 & 1.5688 & YES & YES \\
\hline & $\{2,3,5\}$ & $C S I_{5} \prec C S I_{3} \prec C S I_{1} \prec C S I_{4} \prec C S I_{2}$ & 5.6201 & 1.8734 & YES & YES \\
\hline & $\{2,4,5\}$ & $\mathbf{C S I}_{5} \prec \mathbf{C S I}_{3} \prec \mathbf{C S I}_{1} \prec \mathbf{C S I}_{2} \prec \mathbf{C S I}_{4}$ & 8.4579 & 2.8193 & YES & YES \\
\hline & $\{3,4,5\}$ & $C S I_{5} \prec C S I_{3} \prec C S I_{1} \prec C S I_{2} \prec C S I_{4}$ & 5.8008 & 1.9336 & YES & YES \\
\hline \multirow{5}{*}{ IV-TS } & $\{1,2,3,4\}$ & $C S I_{5} \prec C S I_{3} \prec C S I_{1} \prec C S I_{2} \prec C S I_{4}$ & 7.4283 & 1.2380 & YES & YES \\
\hline & $\{1,2,3,5\}$ & $C S I_{5} \prec C S I_{3} \prec C S I_{1} \prec C S I_{4} \prec C S I_{2}$ & 9.1152 & 1.5192 & YES & YES \\
\hline & $\{1,2,4,5\}$ & $C S I_{5} \prec C S I_{3} \prec C S I_{1} \prec C S I_{2} \prec C S I_{4}$ & 12.3547 & 2.0591 & YES & YES \\
\hline & $\{1,3,4,5\}$ & $C S I_{5} \prec C S I_{3} \prec C S I_{1} \prec C S I_{2} \prec C S I_{4}$ & 9.2014 & 1.5336 & YES & YES \\
\hline & $\{2,3,4,5\}$ & $C S I_{5} \prec C S I_{3} \prec C S I_{1} \prec C S I_{2} \prec C S I_{4}$ & 10.5236 & 1.7539 & YES & YES \\
\hline V-TS & $\{1,2,3,4,5\}$ & $C S I_{5} \prec C S I_{3} \prec C S I_{1} \prec C S I_{4} \prec C S I_{2}$ & 14.4659 & 1.4465 & YES & YES \\
\hline
\end{tabular}


Table 7 shows the overall CSI precedence relations obtained from all 26 possible training sets. The first column in the table shows the category of the TS, i.e. the number of vehicle models involved. Actual optimized values for the objective function in (10) are shown in the fourth column. The fifth column shows the scaled objective values obtained by dividing the actual objective value by the number of summation terms in the objective function. This scaled value is a measure of how well the corresponding CSI model differentiates between the vehicle model pairs.

It is to be noted that 24 out of 26 training sets yielded the correct CSI ordering against (11). From (11) it is seen that Models 2 and 4 have approximately similar ratings, hence the relative positions of $C S I_{2}$ and $C S I_{4}$ are ignored in the table when determining the correctness of the CSI precedence relations. Two training sets in II-TS category (highlighted in Table 7) yielded unsatisfactory results (incorrect CSI ordering or infeasible CSI model): an indication that two vehicle models as a training set may be not be sufficient for generalizing the obtained CSI model for other vehicle models. On the other hand, four or five training sets involve larger datasets and therefore high computational cost. Based on these simple observations we conclude that three training sets are sufficient for generalization in this study. Even within the III-TS category, we find that TS $\{2,4,5\}$ gives the best scaled objective value. Therefore, we shall use $C S I_{\{2,4,5\}}$ in the later sections to show some applications of the proposed CSI modeling technique. Its functional form is given by,

$$
\begin{aligned}
\operatorname{CSI}_{\{2,4,5\}} & =T_{1} \times T_{2} \times T_{3} \times T_{4} \times T_{5} \times T_{6} \\
& =X_{1}^{0.0665} X_{2}^{1.9547} X_{3}^{5.9082} X_{4}^{0.5356} X_{5}^{2.6382} X_{6}^{5.9102}
\end{aligned}
$$

\subsubsection{A Note on Hypothesis Testing}

For the 24 training sets that yielded the correct CSI ordering, the Welch's $t$-test is performed on all vehicle model pairs. The null hypothesis is the same as in (14). However, regardless of the number of vehicle models used, this null hypothesis is tested on all possible model pairs $\left({ }^{5} C_{2}=10\right)$. Thus, a total of 240 tests are performed. We are able to reject the null hypothesis in 239 cases. The failed test case corresponds to $\operatorname{TS}\{2,3\}$ : another indication against using a training set with two vehicle models. The analysis is same as that described in Section 6.2 and hence is omitted here for brevity.

Table 7 also shows the results of the Tukey-Kramer multiple comparison tests. In case of $\operatorname{TS}\{2,3\}$, the hypothesis could not be rejected for the model pair $\{2,3\}$. This agrees with Welch's $t$-test result above. The TukeyKramer test also fails to reject the null hypothesis for the model pair $\{1,2\}$ 
for $\operatorname{TS}\{2,5\}$. This means that the CSI model obtained using the training models 2 and 5 is not adequate to correctly establish the precedence of vehicle models 1 and 2 . The Welch $t$-test failed to identify this issue because of the Type I error discussed previously. For all other training sets, the results of both the significance tests agree as shown in the last two columns of Table 7 .

\subsection{Sensitivity Analysis}

An important part of CRM is to study what factors affect customer satisfaction to what extent. Understanding this gives crucial insights for attracting new customers and retaining old ones. In mathematical terms this study is referred to as sensitivity analysis. In this section we investigate the sensitivity of the CSI models obtained in the III-TS, IV-TS and V-TS categories of Table 7 to the transformed features $X_{i}$. Mathematically, the partial derivative

$$
S_{i}=\frac{\partial f}{\partial X_{i}}
$$

gives the local sensitivity with respect to the $i$-th transformed feature, where $f$ is the functional form of the CSI model under consideration.

In Section 1 we hypothesized that the satisfaction distribution is composed of (two or) three nearly normal distributions of (two or) three statistically differing populations - dissatisfied, satisfied and extremely satis-

fied customers. Here, we are specifically interested in how important these transformed features are for the extremely satisfied set of customers because ultimately they are responsible for the affective and behavioral components of satisfaction, which cannot be measured in quantitative terms. To extend the study over the entire market we choose the top 10\% customers (by CSI value) of Model 2, one of the best vehicle models among the five considered in this paper, thus identifying the extremely satisfied customer set in the market. The sensitivities $S_{i}$ are averaged over this customer set to get $\bar{S}_{i}$ for all 16 CSI models in III-TS, IV-TS and V-TS categories of Table 7. The average sensitivities are ordered according to their decreasing numeric values in Table 8 and classified into two groups of high sensitivity $(\mathrm{H})$ and low sensitivity (L).

The frequency of occurrence of the transformed features (with respect to which the average sensitivities are calculated) in groups $\mathrm{H}$ and $\mathrm{L}$ are shown in Figure 6. The following can be inferred from the figure:

(a) For the majority of extremely satisfied customers, the features $X_{3}$ (total cost), $X_{5}$ (average miles between visits) and $X_{6}$ (sum of severity rat- 
Table 8: Ordering of average CSI model sensitivities $\left(\bar{S}_{i}\right)$ for top $10 \%$ customers (by CSI value) of Model 2. The high sensitivity $(\mathrm{H})$ groups and low sensitivity (L) groups are also shown.

\begin{tabular}{|c|c|c|}
\hline Tra & CSI & Avg. Sensitivity Or \\
\hline Set $(\mathrm{TS})$ & Model & Group H \\
\hline$\{1,2,3\}$ & $C S I_{\{1,2,3\}}$ & $\bar{S}_{3}>\bar{S}_{5}>\bar{S}_{1}>\bar{S}_{6}>\bar{S}_{4}>\bar{S}_{2}$ \\
\hline$\{1,2,4\}$ & $C S I_{\{1,2,4\}}$ & $\bar{S}_{5}>\bar{S}_{1}>\bar{S}_{2}:>\bar{S}_{4}>\bar{S}_{6}>\bar{S}_{3}$ \\
\hline$\{1,2,5\}$ & $C S I_{\{1,2,5\}}$ & $\bar{S}_{3}>\bar{S}_{1}>\bar{S}_{5}>\bar{S}_{6}>\bar{S}_{2}>\bar{S}_{4}$ \\
\hline$\{1,3,4\}$ & $C S I_{\{1,3,4\}}$ & $\bar{S}_{3}>\bar{S}_{6}>\bar{S}_{5}>\bar{S}_{2}>\bar{S}_{4}>\bar{S}_{1}$ \\
\hline$\{1,3$ & $C S I_{\{1,3,5\}}$ & $\bar{S}_{5}>\bar{S}_{1}>\bar{S}_{2}>\bar{S}_{3}>\bar{S}_{6}>\bar{S}_{4}$ \\
\hline$\{1$, & $C S I_{\{1,4,5\}}$ & $\bar{S}_{6}>\bar{S}_{3}>\bar{S}_{5}>\bar{S}_{2}>\bar{S}_{1}>\bar{S}_{4}$ \\
\hline$\{2$, & $C S I_{\{2,3,4\}}$ & $\bar{S}_{6}>\bar{S}_{3}>\bar{S}_{2}:>\bar{S}_{5}>\bar{S}_{4}>\bar{S}_{1}$ \\
\hline$\{2$, & $C S I_{\{2,3,5\}}$ & $\bar{S}_{2}>\bar{S}_{5}>\bar{S}_{3}>\bar{S}_{1}>\bar{S}_{4}>\bar{S}_{6}$ \\
\hline$\{2$, & $C S I_{\{2,4,5\}}$ & $\bar{S}_{3}>\bar{S}_{6}>\bar{S}_{5}>\bar{S}_{2}>\bar{S}_{4}>\bar{S}_{1}$ \\
\hline$\{3,4$ & $C S I_{\{3,4}$ & $\bar{S}_{6}>\bar{S}_{2}>\bar{S}_{3}>\bar{S}_{5}>\bar{S}_{4}>\bar{S}_{1}$ \\
\hline$\{1,2$, & $C S I_{\{1}$, & $\bar{S}_{6}>\bar{S}_{3}>\bar{S}_{5}>\bar{S}_{4}>\bar{S}_{2}>\bar{S}_{1}$ \\
\hline$\{1,2$ & $C S I_{\{1,}$ & $\bar{S}_{1}>\bar{S}_{5}>\bar{S}_{3}:>\bar{S}_{6}>\bar{S}_{4}>\bar{S}_{2}$ \\
\hline$\{1,2$ & $C S I_{\{1,2}$ & $\bar{S}_{6}>\bar{S}_{3}>\bar{S}_{5}>\bar{S}_{2}>\bar{S}_{1}>\bar{S}_{4}$ \\
\hline$\{1,3$, & $C S I_{\{1,3,}$ & $\bar{S}_{2}>\bar{S}_{5}>\bar{S}_{6}>\bar{S}_{3}>\bar{S}_{4}>\bar{S}_{1}$ \\
\hline$\{2,3,4,5\}$ & $C S I_{\{2,3,4,5\}}$ & $\bar{S}_{2}>\bar{S}_{6}>\bar{S}_{5}:>\bar{S}_{3}>\bar{S}_{1}>\bar{S}_{4}$ \\
\hline$\{1,2,3,4,5\}$ & $C S I_{\{1,2,3,4,5\}}$ & $\bar{S}_{6}>\bar{S}_{5}>\bar{S}_{4}>\bar{S}_{3}>\bar{S}_{2}>\bar{S}_{1}$ \\
\hline
\end{tabular}

ings) are the most important factors governing their perception towards vehicle quality and reliability.

(b) The features $X_{1}$ (total number of visits) and $X_{2}$ (total waiting time of customer during vehicle service) are the next most important factors.

(c) The feature $X_{4}$ (average number of days between successive visits) is the least important factor for extremely satisfied customers.

Identification of relative importance of different features in the perception of a customer as outlined above remains as valuable information to designers. This information can be used by designers to prioritize design decisions. For example, improvements to design decisions which are directly 


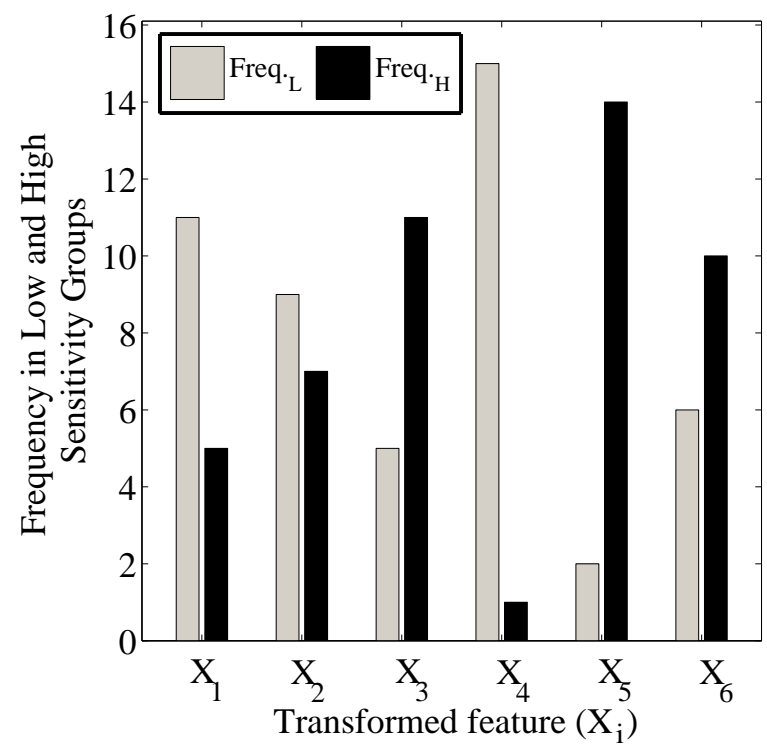

Figure 6: Bar graph showing the frequencies Freq ${ }_{L}$ and Freq $_{H}$ with which the average sensitivity with respect to feature $X_{i}$ occurs in $\mathrm{L}$ and $\mathrm{H}$ groups respectively.

related to features in the H-group of a vehicle model should be given preference over design decisions that affect features belonging to the L-group.

\section{Applications of CSI Model}

In this section, we discuss two applications of the quantitative CSI models which are significant for a customer relations manager to take progressive decisions regarding design enhancements and future market strategy for all five vehicle models. Each application is accompanied by illustrative examples on one or more vehicle models. It is to be noted that similar analyses can be performed on any other vehicle model in a similar manner. However, for the reasons mentioned in Section 6.3, we use the CSI model given in (17) obtained using $\operatorname{TS}\{2,4,5\}$ for all analyses.

\subsection{Application 1: Deriving Classification Rules}

Using a quantitative CSI model and classification tree learning algorithms, we can identify a set of rules to clearly distinguish between any two sets of customers. Identification of such rules for classifying dissatisfied and 
satisfied customers is of prime importance for making future design enhancements to the product. More importantly, this technique allows automobile companies to make buy-back offers to individuals in the dissatisfied set for ensuring customer retention and increasing customer loyalty. The procedure is illustrated here for Model 2. Consider Figure 7, which is a schematic representation of the CSI distribution for Model 2 obtained using $C S I_{\{2,4,5\}}$ in (17).

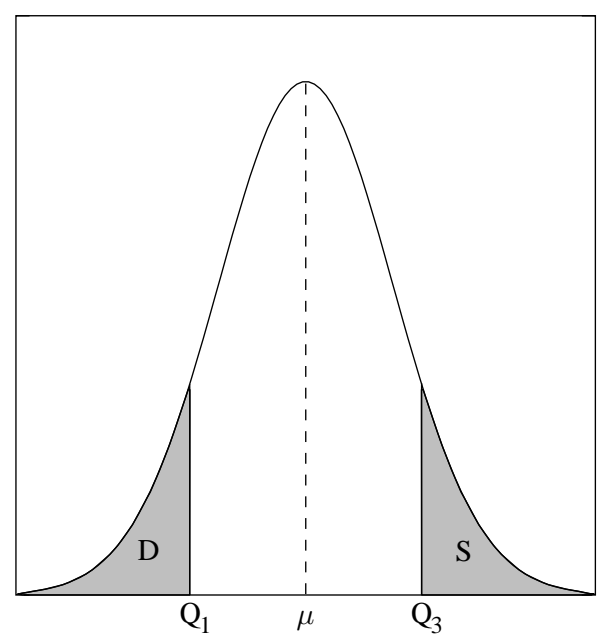

Figure 7: A schematic of the CSI distribution for Model 2 showing regions of satisfied $(S)$ and dissatisfied $(D)$ customers. $Q_{1}$ and $Q_{3}$ represent the first and the third quartiles respectively.

The customers are divided into four equal groups ${ }^{3}$. Customers with CSI rating below the first quartile $Q_{1}$ are labeled as Dissatisfied (Class $D)$ and those with CSI rating above the third quartile $Q_{3}$ are labeled as Satisfied (Class $S$ ). Matlab's classregtree algorithm is used to generate the classification tree in Figure 8. The features $x_{i}$ extracted in Section 3 are used as predictors for classifying customers as either belonging to $D$ or $S$. The transformed features are not used here since their values are normalized and are of no relevance to a practitioner. At the root node of the classification tree we have equal number of $S$ and $D$ customers. The algorithm optimally branches a node such that at each sub-node the number of customers from one class $(D$ or $S$ ) decreases and those from the other

\footnotetext{
${ }^{3}$ This is for illustration only. The actual choice depends on the purpose of the analysis.
} 
class increases. The process is continued until the number of customers from either of the two classes falls below $1 \%$. This procedure is called pruning.

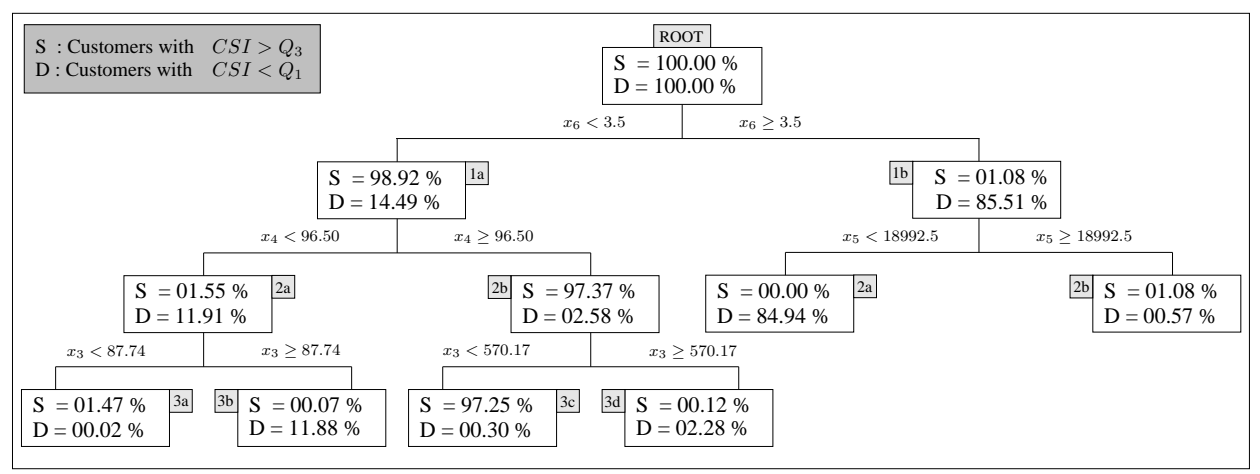

Figure 8: Classification tree for satisfied and dissatisfied customers of Model 2.

The classification tree reveals some very interesting rules for classifying satisfied and dissatisfied customers:

Rule-I: $97.25 \%$ customers from Class $S$ follow the nodal path: ROOT $\rightarrow$ $1 \mathrm{a} \rightarrow 2 \mathrm{~b} \rightarrow 3 \mathrm{c}$. This means almost all customers for whom,

$$
\left[x_{6}<3.5 \text { AND } x_{4} \geq 96.5 \text { AND } x_{3}<570.17\right] \text { are satisfied. }
$$

Rule-II: $85.51 \%$ customers from $D$ follow the nodal path: ROOT $\rightarrow 1 \mathrm{~b}$. Another $11.88 \%$ customers from $D$ follow the path: ROOT $\rightarrow 1 \mathrm{a} \rightarrow 2 \mathrm{a} \rightarrow 3 \mathrm{~b}$. Thus for $97.39 \%(85.51 \%+11.88 \%)$ of dissatisfied customers,

$$
\left[\left(x_{6} \geq 3.5\right) \text { OR }\left(x_{6}<3.5 \text { AND } x_{4}<96.5 \text { AND } x_{3} \geq 87.74\right)\right] .
$$

Rule-I says that for the period considered in this study, if the following:

1. Sum of severity ratings of all repairs to the customer's vehicle is less than 3.5,

2. Average number of days between successive customer visits is more than 96.5 days, and

3. Total cost of repairs for the entire repair period is less than $\$ 570.17$.

are all true for a customer, then it is very likely that he/she is satisfied with Model 2.

Similarly, Rule-II says that a customer is very likely to be dissatisfied with Model 2 if either, 
1. Sum of severity ratings of all repairs to the customer's vehicle is more than 3.5,

or all of the following are true:

1. Sum of severity ratings of all repairs to the customer's vehicle is less than 3.5,

2. Average number of days between successive customer visits is less than 96.5 and

3. Total cost of repairs for entire service period is more than $\$ 87.74$.

Clearly, such classification rules carry a lot of significance in data mining exercises relevant to CRM applications.

\subsection{Application 2: Identification of Critical Field Failures}

A large customer base is also associated with a large number of repair types or failures. Table 1 shows the number of unique field failures in the five vehicle models considered. For planning future design enhancements, it becomes necessary to identify critical failures and deemphasize insignificant ones. Usually, this decision is based on the frequency or severity of the failures and on market feedback. In this section, we propose a method for prioritizing different field failures on the basis of the percentage improvement in overall satisfaction that can be achieved by eliminating (or reducing) their occurrence through design improvements. We call this quantity the CSI Improvement Potential (CIP) value of the field failure regardless of whether the corresponding field failure is completely or partially removed.

\subsubsection{Procedure for Evaluating CIP}

Let $\mathbf{C S I}_{\text {orig }}$ be the $C \times 1$ column vector of unnormalized CSI values obtained using any of the CSI models (say $C S I_{\{.\}}$) discussed in Section 6.3 for any given vehicle model with $C$ customers. Then the procedure for calculating the CIP for $100 \%$ reduction of failures with repair code $r_{c}$ is as follows:

Step 1: Remove all claims (for $100 \%$ reduction) containing repair code $r_{c}$ from the service dataset. Some customers with only failures corresponding to $r_{c}$ will be deleted altogether in the process. Let the number of such customers be $Z$. We will later compensate this reduction in the number of customers.

Step 2: Extract all six features $x_{i}$, discussed in Section 3, to obtain the feature matrix $F M$ of size $(C-Z) \times 6$. It is to be noted that reduction of 
any kind of failure for a customer should improve his/her satisfaction. However, when evaluating $x_{4}$ and $x_{5}$ for customers whose first or last visit has the repair code $r_{c}$, it is possible that the decrease in numerator of Equation (2) and/or Equation (3) is numerically greater than the decrease in denominator, thus decreasing the CSI value instead of increasing it. To avoid this, the numerator is not changed.

Step 3: Compensate for the deleted customers (who are now problem free) by adding $Z$ instances of hypothetical customers with feature vector $\left[\min \left(x_{1}\right) \min \left(x_{2}\right) \min \left(x_{3}\right) \max \left(x_{4}\right) \max \left(x_{5}\right) \min \left(x_{6}\right)\right]$ to $F M$. This ensures that these customers are the most satisfied among the $C$ customers.

Step 4: Normalize columns of $x_{i}$ 's in $F M$ (now of size $C \times 6$ ) as described in Section 4 to get the transformed feature matrix $F M^{t r}$.

Step 5: Obtain $\mathbf{C S I}_{n e w}$, a $C \times 1$ column vector of unnormalized CSI values, using $C S I_{\{.\}}$on $F M^{t r}$.

Step 6: Normalize the $2 C \times 1$ sized column vector $\mathbf{C S I}=\left[\mathbf{C S I}_{\text {orig }} \mathbf{C S I}_{\text {new }}\right]^{T}$ between 0 and 1 using $\min (\mathbf{C S I})$ and $\max (\mathbf{C S I})$ to obtain $\mathbf{C S I}^{n r}=$ $\left[\mathbf{C S I}_{\text {orig }}^{n r} \mathbf{C S I}_{\text {new }}^{n r}\right]^{T}$. This allows unbiased comparison between the original and new CSI distributions.

Step 7: The CIP for 100\% reduction of failures with repair code $r_{c}$ can now be calculated as,

$$
C I P=\frac{\operatorname{mean}\left(\mathbf{C S I}_{\text {new }}^{n r}\right)-\operatorname{mean}\left(\mathbf{C S I}_{\text {orig }}^{n r}\right)}{\operatorname{mean}\left(\mathbf{C S I}_{\text {orig }}^{n r}\right)} \times 100 \% .
$$

\subsubsection{Prioritizing Failures based on CIP}

The ability to calculate CIP allows us to prioritize failures based on their impact on the CSI rating. Rectification of root causes of high priority failures leads to improvement in the design and more importantly improves the customer perception of the product. Traditionally, prioritization is done based on the frequency of occurrence of failures. Here, we investigate whether such an approach really improves the satisfaction proportionately. Models 1 and 5 are chosen for this study as they are the worst rated models in the nonluxury and luxury segments respectively (see Table 1 and Equation (11)). Figure 9 shows bar charts of CIP values corresponding to $100 \%$ removal of the top 50 most frequently occurring repair codes or failures (arranged in decreasing order of frequency along the X-axis) in Models 1 and 5. The 
CIP values are calculated using $C S I_{\{2,4,5\}}$. Note how the assumption that frequency of occurrence is directly proportional to the improvement in satisfaction holds good for Model 1 but not for Model 5. A viable approach for

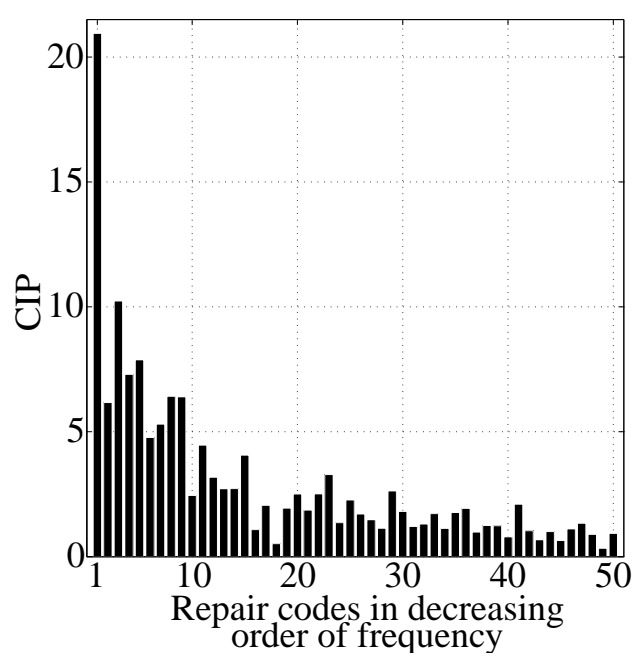

(a)

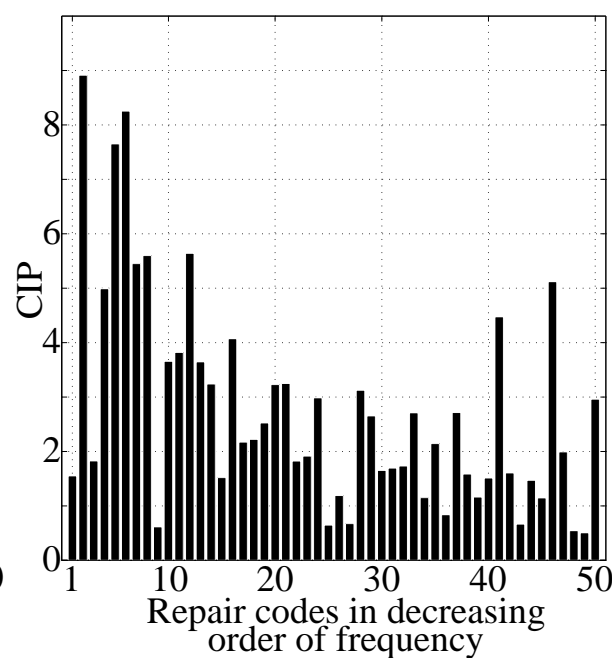

(b)

Figure 9: CIP values corresponding to $100 \%$ removal of the top 50 most frequent repair codes for (a) Model 1 and (b) Model 5.

quantitatively identifying high-priority field failures, like the one presented here, is very useful for making progressive improvements to the vehicle design while ensuring that the customers are maximally satisfied with each design change.

\subsubsection{CIP for Partial Reduction of Failures}

Although the highest improvement in customer satisfaction is achieved by the complete removal of high-priority field failures, it may not always be practical. In such cases, it may be useful to study the effect of partial reduction of failures. Important insights into the nature of CIP can be gained in the process. Model 1 is used here for illustrating the CIP behavior with respect to the most frequent repair code. Again the CSI model $C S I_{\{2,4,5\}}$ is used for all CIP calculations. Figure 9(a) reveals that the complete removal of the most frequent repair code leads to around $20 \%$ improvement in satisfaction. On the other hand, Figure 10 shows the progressive improvement in satisfaction for every $10 \%$ additional removal of the most frequent repair code, starting from $0 \%$ (where obviously $C I P=0 \%$ ) to $100 \%$ (where 


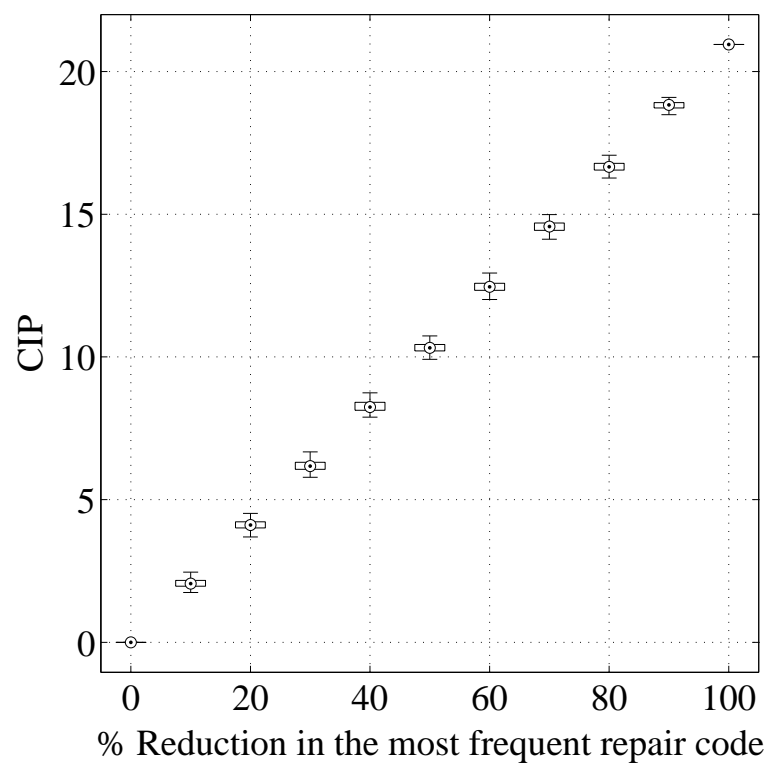

Figure 10: Box plot of CIP values for every $10 \%$ partial reduction of the most frequent repair code in Model 1.

$C I P \approx 20 \%$ as seen in Figure $9(\mathrm{a}))$. Figure 10 is generated using a sampling method. For CIP calculation of any intermediate $p \%$ removal, a sample of 100 datasets is first created by randomly removing $p \%$ of the most frequent repair code. Thereafter, the CIP is calculated for all 100 datasets and a corresponding box-whisker ordinate is plotted at $p \%$ abscissa. The lower and upper edges of rectangular box represents the first and the third quartiles respectively of the 100 CIP values, the circle in the rectangular box represents their median value, and the whiskers represent the extreme CIP values.

\subsubsection{Partial Reduction Plans}

Figure 11 shows the CIP values for every $10 \%$ partial reduction of the top five most frequent repair codes (named $r_{1}$ through $r_{5}$ ) occurring in Model 1. The plots are obtained using exactly the same procedure as described above except that only the median values are shown for the box-whisker ordinates for clarity. It can be verified that the CIP values at $100 \%$ reduction of each repair code correspond to the first five bars in Figure 9(a). Note that despite using a highly non-linear CSI function given by $C S I_{\{2,4,5\}}$ from (17), the CIP increases approximately linearly with the amount of reduction for all five repair codes. More importantly, note that different repair codes give 
different rates of improvement in satisfaction.

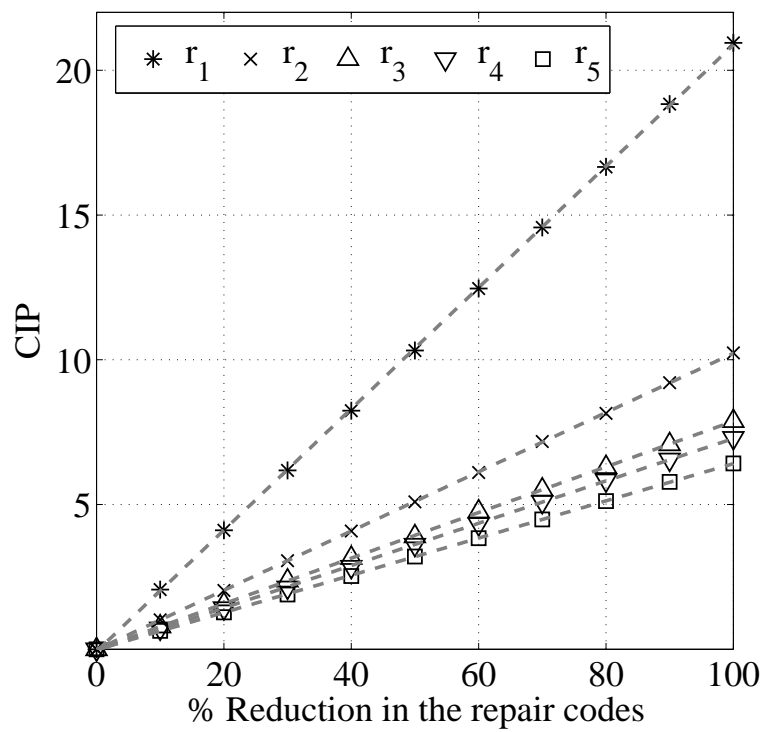

Figure 11: CIP median values for every $10 \%$ partial reduction of the top five most frequent repair codes in Model 1.

With this information in hand, the next question to ask is what combination of repair codes should be used and how much reduction should be targeted for each repair code to achieve a desired level of improvement in customer satisfaction. Typically, the number of repair codes associated with each vehicle model, as shown in Table 1, is very high. For illustration we consider five hypothetical Partial Reduction Plans (PRPs) shown in Table 9 prescribed by experts in the field. For example, PRP-I represents a $10 \%$ reduction in repair code $r_{1}, 20 \%$ in $r_{2}, 30 \%$ in $r_{3}, 20 \%$ in $r_{4}$ and $10 \%$ reduction in $r_{5}$.

Table 9: Five hypothetical Partial Reduction Plans (PRPs) using the top five most frequent repair codes of Model 1.

\begin{tabular}{|c|c|c|c|c|c|}
\cline { 2 - 6 } \multicolumn{1}{c|}{} & PRP-I & PRP-II & PRP-III & PRP-IV & PRP-V \\
\hline$r_{1}$ & $10 \% \downarrow$ & $10 \% \downarrow$ & $10 \% \downarrow$ & $10 \% \downarrow$ & $10 \% \downarrow$ \\
$r_{2}$ & $20 \% \downarrow$ & $10 \% \downarrow$ & $10 \% \downarrow$ & $10 \% \downarrow$ & $20 \% \downarrow$ \\
$r_{3}$ & $30 \% \downarrow$ & $20 \% \downarrow$ & $10 \% \downarrow$ & $10 \% \downarrow$ & $10 \% \downarrow$ \\
$r_{4}$ & $20 \% \downarrow$ & $20 \% \downarrow$ & $20 \% \downarrow$ & $10 \% \downarrow$ & $10 \% \downarrow$ \\
$r_{5}$ & $10 \% \downarrow$ & $20 \% \downarrow$ & $20 \% \downarrow$ & $20 \% \downarrow$ & $10 \% \downarrow$ \\
\hline
\end{tabular}


The sampling method is again used here for calculating the CIP value for each PRP shown in Table 9. Hundred datasets are created by randomly removing the specified percentages of all five repair codes. The corresponding CIP values are calculated as before. The median of these CIP values for all five hypothetical PRPs are shown in the first row of Table 10. We

Table 10: Comparison between median CIP values obtained by the sampling method and by the principle of superposition using Figure 11.

\begin{tabular}{|c|c|c|c|c|c|}
\cline { 2 - 6 } \multicolumn{1}{c|}{} & PRP-I & PRP-II & PRP-III & PRP-IV & PRP-V \\
\hline CIP Median (using 100 samples) & 8.58 & 7.39 & 6.59 & 5.87 & 6.25 \\
\hline CIP Median (from Figure 11) & 8.51 & 7.32 & 6.55 & 5.85 & 6.23 \\
\hline
\end{tabular}

now estimate the CIP values for the same PRPs using Figure 11 by adding the individual CIP values of the five repair codes obtained by the amount of reduction specified by the PRP. For example, in case of PRP-I,

$$
\begin{aligned}
C I P_{P R P-I}= & C I P_{r_{1}}(10 \%)+C I P_{r_{2}}(20 \%)+C P_{r_{3}}(30 \%) \\
& +C I P_{r_{4}}(20 \%)+C I P_{r_{5}}(10 \%) \\
= & 2.059 \%+2.034 \%+2.358 \%+1.426 \%+0.635 \% \\
= & 8.512 \% .
\end{aligned}
$$

This is known as the principle of superposition. The second row of Table 10 shows that this principle approximately holds for CIP calculations with different reduction plans. This is due to the fact that most failure types are independent of each other. The small variation is seen due to the finite number (100) of samples used for CIP calculation.

\section{Conclusions}

In this study, we have demonstrated how starting with vehicle service and sales data, a plethora of valuable information can be derived about perceived customer satisfaction indicators. First, a single-objective optimization procedure has been suggested to develop Customer Satisfaction Index (CSI) models for different vehicle models as a function of six important features extracted from the service data. This customer level CSI modeling approach has given us an understanding of the objective space and difficulties associated with it. Consequently, we developed the multiple vehicle CSI modeling technique through which a single aggregate CSI function can allow us to rank different vehicle models in increasing order of customer satisfaction. 
The ranking of five different vehicle models obtained by our procedure has been statistically validated to be significant and also verified against the ranking obtained from published Consumer Reports reliability ratings on the same set of vehicle models.

The generalizing capability of the proposed optimization approach has been tested by using a subset of vehicle models for the optimization task and testing the remaining vehicle models using the obtained CSI function. For the five vehicle models, the use of a maximum of three during optimization was found to be adequate to correctly rank all five vehicle models. This demonstrates the scalability of the proposed procedure in terms of developing the CSI model by using a few vehicle models and then applying the resulting CSI model to predict CSI values for other vehicle models which were not considered in the developmental phase.

Although the above optimization-based technique for developing a mathematical CSI function is novel and has been shown to be effective on a fivevehicle database, another equally significant contribution of this study comes from two post-processing applications. The developed and tested CSI function can be used to group customers into different classes, of which 'satisfied' and 'dissatisfied' customers can be isolated for further knowledge-gathering actions. For a particular vehicle model, we have identified the relative importance of different warranty-related features. It has been observed that total cost of repair, average miles between visits and the sum of severity ratings of repairs are the most important features causing the perceived satisfaction of a customer. Contrary to general belief, average number of days between successive visits has been found to be the least important factor influencing customer satisfaction. By identifying the extremely satisfied customers and dissatisfied customers from the obtained CSI function for a vehicle model, classification rules relating to the features that are responsible for influencing the customers have been obtained by using a classification tree algorithm. These rules can help a company in easily identifying dissatisfied customers for making buy-back or other customer-winning proposals.

Automobile industries are also interested in identifying field failures that are critically responsible for causing customer dissatisfaction. If such critical field failures can be identified, an effort to reduce them through future design improvements would be an indirect benefit of the CSI study. With the developed CSI function model, a number of field failures have been considered for this purpose. First, our analysis has identified a set of critical field failures that most significantly affect the CSI function and then we have demonstrated how a partial or complete removal of such critical field failures can lead to improvement in perceived customer satisfaction. All the 
above analyses, originating from the proposed CSI modeling approach, have been shown to provide useful insights for improving perceived customer satisfaction. But in the long run such information should also indirectly help in designing a better product.

Our study clearly demonstrates the usefulness of after-sales service and warranty data in four viable ways in the design cycle: (i) rank and reveal a perceived level of customer satisfaction index for a platform of products, (ii) identify different data features and their combinations that are responsible for establishing a high (or low) customer satisfaction index, (iii) identify satisfied and dissatisfied customers for further action through customer relationship management programs, and (iv) rank different field failure events according to their criticality for a possible improvement in the customer satisfaction index of a product. Since after-sale data speaks volumes about a product's direct operational performance and its perception by real customers, the above analysis, when performed with a larger volume of customer data, is likely to bring out valuable and realistic information. The methodologies discussed in this paper are generic and can be applied to other consumer product design and development problems.

\section{Acknowledgments}

The financial support and vehicle related data provided by India Science Lab, General Motors R\&D are greatly appreciated. Authors thank Dr. Prakash G. Bharati and Dr. Pattada A. Kallappa for helpful discussions.

\section{References}

[1] ISO 9001:2008: Quality management systems - Requirements, ISO/TC 176 (2008).

[2] C. Cianfrani, J. Tsiakals, J. West, ISO 9001: 2008 Explained, ASQ Quality Press, 2009.

[3] L. Dube, K. Menon, Multiple roles of consumption emotions in postpurchase satisfaction with extended service transactions, International Journal of Service Industry Management 11 (3) (2000) 287-304.

[4] L. Berry, Marketing services: Competing through quality, Free Press, 2004. 
[5] S. Kessler, Customer satisfaction toolkit for ISO 9001: 2000, American Society for Quality, 2002.

[6] R. Likert, A technique for the measurement of attitudes, New York: Columbia University Press, 1932.

[7] S. Eroglu, K. Machleit, An empirical study of retail crowding: Antecedents and consequences, Journal of Retailing 66 (2) (1990) 201-221.

[8] R. Westbrook, A rating scale for measuring product/service satisfaction, The Journal of Marketing (1980) 68-72.

[9] The American Customer Satisfaction Index, ACSI, www.theacsi.org (2010).

[10] JDPower.com, J.D. Power and Associates, www.jdpower.com (2010).

[11] ConsumerReports.org, Consumers Union of U.S., Inc., www.consumerreports.org (2010).

[12] J. Robinson, S. Chukova, Estimating mean cumulative functions from truncated automotive warranty data, in: Communications of the Fourth International Conference on Mathematical Methods in Reliability, Methodology and Practice, Santa Fe, New Mexico, USA, 2004, pp. CD-ROM (4 pages).

[13] A. Parasuraman, V. Zeithaml, L. Berry, A conceptual model of service quality and its implications for future research, The Journal of Marketing (1985) 41-50.

[14] A. Parasuraman, V. Zeithaml, L. Berry, SERVQUAL: A multiple-item scale for measuring consumer perceptions of service quality, Journal of Retailing 64 (1) (1988) 12-40.

[15] S. Wu, Warranty data analysis: a review, Quality and Reliability Engineering International 28 (8) (2012) 795-805.

[16] S. Bandaru, K. Deb, V. Khare, R. Chougule, Quantitative modeling of customer perception from service data using evolutionary optimization, in: Proceedings of the 13th annual conference on Genetic and evolutionary computation, ACM, 2011, pp. 1763-1770.

[17] J.D. Power/What Car? 2009 UK Vehicle Ownership Satisfaction Study, www.testdriven.co.uk (2009). 
[18] N. Anisor, D. Adela-Eliza, C. Luciana, The method used for measuring the customers' satisfaction, in: Proc. 9th WSEAS International Conference on Education And Educational Technology (EDU'10), 2010, pp. 197-200.

[19] J. Koza, Genetic programming: On the programming of computers by means of natural selection, MIT press, 1992.

[20] K. Deb, A. Pratap, S. Agarwal, T. Meyarivan, A fast and elitist multiobjective genetic algorithm: NSGA-II, IEEE Transactions on Evolutionary Computation 6 (2) (2002) 182-197.

[21] K. Deb, R. Agrawal, Simulated binary crossover for continuous search space, Complex Systems 9 (2) (1995) 115-148.

[22] K. Deb, Multi-objective optimization using evolutionary algorithms, Wiley, Chichester, UK, 2001.

[23] K. Deb, S. Gupta, Understanding knee points in bicriteria problems and their implications as preferred solution principles, Engineering optimization 43 (11).

[24] ConsumerReports, ConsumersReports.org (April 2010).

[25] K. Deb, An efficient constraint handling method for genetic algorithms, Computer Methods in Applied Mechanics and Engineering 186 (2-4) (2000) 311-338.

[26] R. Lomax, An introduction to statistical concepts for education and behavioral sciences, Lawrence Erlbaum, 2001.

[27] J. Hsu, Multiple comparisons: theory and methods, CRC Press, 1996. 